\title{
ON THE GENERATION OF ONE-RELATOR GROUPS $\left({ }^{1}\right)$
}

\author{
BY
}

\section{STEPHEN J. PRIDE}

\begin{abstract}
This paper is concerned with obtaining information about the Nielsen equivalence classes and $T$-systems of certain two-generator $H N N$ groups, and in particular of certain two-generator one-relator groups. The theorems presented here extend results of the author appearing in the Proceedings of the Second International Conference on the Theory of Groups. In particular it is shown here that if $G=\left\langle a, t ; a^{\alpha} E_{r}^{-1} a^{\beta} E_{r} \ldots a^{\alpha} s_{r}^{-1} a^{\beta} s_{r}\right)$ where the $\alpha_{j}$ are positive, the $\beta_{i}$ are nonzero, $E_{r}$ has the form $\left[a^{\epsilon_{1}},\left[a^{\epsilon},\left[\cdots,\left[a^{\epsilon}, t\right] \cdots\right]\right]\right]$ with $\left|\epsilon_{1}\right|=$ $\left|\epsilon_{2}\right|=\cdots=\left|\epsilon_{r}\right|=1$, then in a large number of cases $G$ has one Nielsen equivalence class. Similar results are also obtained for certain groups with more than one relator. A fair proportion of the paper is given to developing a method for reducing pairs of elements in $H N N$ groups. This method has some of the features of Nielsen's reduction theorem for free groups. One other interesting result obtained here is that a one-relator group with torsion which has one $T$-system is Hopfian. The early part of the paper is discursive. It contains most of the known results concerning $T$-systems of one-relator groups, and highlights several open problems, some of which have been raised by other authors.
\end{abstract}

The paper is divided into three sections. The first section is fairly informal and contains all the main results. The second section is devoted to proving two reduction theorems used in $\S 1$. The third section is given to establishing that various groups satisfy conditions enumerated in $\S 1$. (These conditions ensure that one can determine the Nielsen equivalence classes of certain $H N N$ extensions of the groups in question.) Each section is subdivided, and has a short introduction explaining its contents more fully.

The standard reference for notation and background material used throughout this paper will be the book [12] by Magnus, Karrass and Solitar. Additional concepts and notation will be needed as follows. Let $G$ be a group. If $A$ is a sub-

Received by the editors July 17, 1974.

AMS (MOS) subject classifications (1970). Primary 20F05, 20F10; Secondary $20 \mathrm{E} 05$.

Key words and phrases. One-relator group, Nielsen equivalence, $T$-system, isomorphism problem, Hopfian, $H N N$ group, $t$-reduced and cyclically $t$-reduced words, Britton's Lemma, malnormal subgroup, two-generator problem, small cancellation group, Fuchsian group.

(1) The early part of this work was done at the Australian National University with the support of an Australian Commonwealth Postgraduate Award, as part of the author's Ph. D. thesis written under Dr. M. F. Newman. The latter part of the work was carried out at the Open University, England. The author is grateful to Dr. A. M. Brunner for many interesting discussions. 
set of $G$ then sgp $A$ will denote the subgroup of $G$ generated by $A$. An ordered $r$-tuple $\left(g_{1}, g_{2}, \ldots, g_{r}\right)$ of elements of $G$ will be said to generate $G$, or to be a generating $r$-tuple of $G$, if $\operatorname{sgp}\left\{g_{1}, g_{2}, \ldots, g_{r}\right\}=G$.

Suppose $G=\langle a, b, c, \ldots ; P, Q, R, \ldots\rangle$, and let $W, W^{\prime}$ be words in the generators $a, b, c, \ldots$ of $G$. Then $W \equiv W^{\prime}$ will mean that $W$ and $W^{\prime}$ are the same word. If $W$ and $W^{\prime}$ define the same element of $G$ then it will be said that $W$ is equal to $W^{\prime}$ in $G$, written $W=W^{\prime}$ in $G$ (or simply $W=W^{\prime}$ if $G$ is understood). The generator $a$ of $G$ will be said to occur to exponent $e$ in $W$ if $W \equiv S a^{e} T$ where neither the last symbol of $S$ nor the first symbol of $T$ is $a$ or $a^{-1}$. If $a$ occurs to an exponent different from zero in $W$ then it will be said that $W$ involves $a$, or equivalently that $a$ occurs in $W$. The number of symbols in the word $W$ will be called the length of $W$ and be denoted by $L(W)$. A word $V$ will be said to be more than half of $W$ if $V$ is a subword of $W$ and $L(V)>L(W) / 2$. Concepts such as more than $5 / 6$, exactly half etc. are defined in an analogous manner.

When a group $G$ is given in terms of generators $a, b, c, \ldots$ and defining relators, words in $a, b, c, \ldots$ will often be identified with the elements of $G$ which they represent. The context should make it clear when this identification is being made. In particular, it will nearly always be made if $G$ is free on $a, b, c$, .... In this case the length of an element of $G$ will be taken to be the length of the unique freely reduced word defining that element.

The functions [ ] and sg will be needed. These are defined for a real number $\nu$ as follows: $[\nu]$ is the greatest integer less than or equal to $\nu$;

$$
\operatorname{sg}(\nu)=\left\{\begin{aligned}
-1 & \text { if } \nu<0 \\
0 & \text { if } \nu=0 \\
1 & \text { if } \nu>0
\end{aligned}\right.
$$

Throughout the paper $\epsilon$ (or some variation such as $\epsilon^{\prime}, \epsilon_{1}, \epsilon_{2}$ ) will denote an integer of modulus 1 .

1. T-systems of one-relator groups. There is a certain way of partitioning the generating $r$-tuples of a group which is useful when considering the isomorphism and other problems. This partition is discussed in $\S 1.1$, with particular reference to one-relator groups. It is shown that if $G$ is a one-relator group on $r$-generators having only one element in the partition then the isomorphism problem is solvable for $G$ in various instances. Moreover, if $G$ has torsion then $G$ is Hopfian. Some open problems are also raised in $\S 1.1$.

In $\S 1.2$ a theorem is obtained which enables one to calculate the number of elements in the partition of the generating pairs of certain two-generator groups. This theorem is then applied in $\S \S 1.3,1.4$, paying particular attention to onerelator groups. 
1.1 On T-systems. Let $r$ be a positive integer and let $G$ be an $r$-generator group. Two generating $r$-tuples

$$
\mathbf{g}=\left(g_{1}, g_{2}, \ldots, g_{r}\right) \text { and } \mathbf{g}^{\prime}=\left(g_{1}^{\prime}, g_{2}^{\prime}, \ldots, g_{r}^{\prime}\right)
$$

are said to be Nielsen equivalent if there is an automorphism

$$
x_{i} \rightarrow Y_{i}\left(x_{1}, x_{2}, \ldots, x_{r}\right), \quad i=1,2, \ldots, r,
$$

of the free group $F_{r}$ on $x_{1}, x_{2}, \ldots, x_{r}$ such that $g_{i}^{\prime}=Y_{i}\left(g_{1}, g_{2}, \ldots, g_{r}\right)$ for $i=1,2, \ldots, r$. The $r$-tuples $\mathbf{g}$ and $\mathbf{g}^{\prime}$ are said to lie in the same $T$-system [19] if there is an automorphism $\rho$ of $G$ such that $\mathbf{g}^{\prime}$ is Nielsen equivalent to $\rho \mathbf{g}=$ $\left(\rho\left(g_{1}\right), \rho\left(g_{2}\right), \ldots, \rho\left(g_{r}\right)\right)$.

The concept of a $T$-system is of importance in classifying the different $r$-generator presentations of $G$, and therefore has some bearing on the isomorphism and related problems. Let $\left\langle x_{1}, x_{2}, \ldots, x_{r} ; R_{1}, R_{2}, \ldots, R_{m}\right\rangle$ be a presentation of the group $G$ associated with the $r$-tuple $g$. (That is, the kernel of the homomorphism of $F_{r}$ onto $G$ defined by $x_{i} \rightarrow g_{i}$ for $i=1,2, \ldots, r$ is the normal subgroup of $F_{r}$ generated by $\left\{R_{1}, R_{2}, \ldots, R_{m}\right\}$.) Then it is not difficult to show that $\mathbf{g}^{\prime}$ is in the same $T$-system as $\mathbf{g}$ if and only if there is an automorphism $\psi$ of $F_{r}$ such that $\left\langle x_{1}, x_{2}, \ldots, x_{r} ; \psi\left(R_{1}\right), \psi\left(R_{2}\right), \ldots, \psi\left(R_{m}\right)\right\rangle$ is a presentation of $G$ associated with $\mathbf{g}^{\prime}$. This does not in general imply that the relators in every $m$-relator presentation $\left\langle x_{1}, x_{2}, \ldots, x_{r}, S_{1}, S_{2}, \ldots, S_{m}\right\rangle$ of $G$ associated with $\mathbf{g}^{\prime}$ are very closely connected to the words $\psi\left(R_{1}\right), \psi\left(R_{2}\right), \ldots, \psi\left(R_{m}\right)$. However, in the special case when $m=1$ it follows from the Conjugacy Theorem for Groups with One Defining Relation [12, p. 261] that $S_{1}$ must be a conjugate of $\psi\left(R_{1}\right)$ or its inverse. Or put another way:

LEMMA 1. Two one-relator presentations $\left\langle x_{1}, x_{2}, \ldots, x_{r} ; R\right\rangle,\left\langle x_{1}, x_{2}\right.$, $\left.\ldots, x_{r} ; S\right\rangle$ of a group $G$ are associated with elements from the same $T$-system of $G$ if and only if $S$ is equal in $F_{r}$ to the image of $R$ or $R^{-1}$ under an automorphism of $F_{r}$.

This fact can be used to solve the isomorphism problem for $G$ in certain instances. The meaning of the isomorphism problem is the following. Let $G=$ $\left\langle a_{1}, a_{2}, \ldots, a_{p} ; P_{1}, P_{2}, \ldots, P_{n}\right\rangle$ where $p$ and $n$ are finite, and let $S$ be a recursive set of finite presentations of groups. The isomorphism problem (IsoP) for $G$ (or more precisely for this presentation of $G$ ) relative to $S$ is the algorithmic problem of deciding for any element of $S$ whether or not it is a presentation of $G$.

THEOREM 1. Let $G=\left\langle x_{1}, x_{2}, \ldots, x_{r} ; R\right\rangle$.

(i) Suppose that if $\left\langle x_{1}, x_{2}, \ldots, x_{r} ; S\right\rangle$ is a presentation of $G$ then 
$\left\langle x_{1}, x_{2}, \ldots, x_{r} ; R\right\rangle$ and $\left\langle x_{1}, x_{2}, \ldots, x_{r} ; S\right\rangle$ are associated with elements from the same $T$-system of $G$. Then IsoP is solvable for $G$ relative to every recursive set of one-relator presentations.

(ii) Suppose that $G$ has one T-system. Assume that there is an element of finite order $n(n>1)$ in $G$, and let $S$ be a recursive set of finite presentations of the form $\left\langle x_{1}, x_{2}, \ldots, x_{r} ; P_{1}, P_{2}, \ldots, P_{m}\right\rangle$. Suppose that there is an algorithm which when applied to any element $\left\langle x_{1}, x_{2}, \ldots, x_{r} ; P_{1}, P_{2}, \ldots, P_{m}\right\rangle$ of $S$ enables one to decide for any word $W$ in $x_{1}, x_{2}, \ldots, x_{r}$ whether or not $W^{n}$ is a consequence of $P_{1}, P_{2}, \ldots, P_{m}$. Then IsoP is solvable for $G$ relative to $S$.

Part (i) of this theorem is well-known. To prove it, first assume that $R$ is empty. Then it follows from Theorem N3 of [12] that $\left\langle x_{1}, x_{2}, \ldots, x_{s} ; S\right\rangle$ is a presentation of $G$ if and only if either $s=r$ and $S$ is empty, or $s=r+1$ and $S$ is a primitive element of $F_{r+1}$. Since there is an algorithm for determining whether an element of a free group is a primitive or not [12, Theorem N2] the result follows in this case. Next suppose that $R$ is primitive. Then again by Theorem N3 of [12], $\left\langle x_{1}, x_{2}, \ldots, x_{s} ; S\right\rangle$ is a presentation of $G$ if and only if either $s=r$ and $S$ is primitive or $s=r-1$ and $S$ is empty, so the result holds in this case (using Theorem N2 of [12]). Finally, suppose $R$ is neither empty nor primitive. Then by Corollary 5.14.2 of [12], Theorem N3 of [12], and Lemma 1 above, $\left\langle x_{1}, x_{2}, \ldots, x_{s} ; S\right\rangle$ is a presentation of $G$ if and only if $s=r$ and $S$ is equal in $F_{r}$ to an automorphic image of $R$ or $R^{-1}$. The solution of the isomorphism problem therefore follows using Theorem N2 of [12].

Now consider part (ii) of the theorem. Since $G$ has elements of order $n$ there is a word $Q$ such that $R \equiv Q^{n}[12, \S 4.4]$. Also, since $G$ has one $T$-system, an element $\left\langle x_{1}, x_{2}, \ldots, x_{r} ; P_{1}, P_{2}, \ldots, P_{m}\right\rangle$ of $S$ is a presentation of $G$ if and only if there is a cyclically reduced word $S$ which is an automorphic image of $Q$ or $Q^{-1}$ such that the normal subgroups of $F_{r}$ generated by $\left\{P_{1}, P_{2}, \ldots, P_{m}\right\}$ and $\left\{S^{n}\right\}$ are the same. Now observe that if the words $P_{1}, P_{2}, \ldots, P_{m}$ (assumed nonempty and freely reduced) are consequences of a cyclically reduced word $S^{n}$ then

$$
L(S)<(n-1)^{-1} \min \left\{L\left(P_{1}\right), L\left(P_{2}\right), \ldots, L\left(P_{m}\right)\right\}
$$

by Newman's Spelling Theorem [20, Theorem 3]. Therefore consider the set $W$ of cyclically reduced words of length less than

$$
(n-1)^{-1} \min \left\{L\left(P_{1}\right), L\left(P_{2}\right), \ldots, L\left(P_{m}\right)\right\},
$$

and let $U$ be the subset of $W$ consisting of those words whose $n$th power is a consequence of $\left\{P_{1}, P_{2}, \ldots, P_{m}\right\}$. By assumption there is an algorithm to determine 
the elements of $U$, and the algorithm is the same no matter which element of $S$ is chosen at the outset. Let

$$
V=\left\{V: V \in U \text {, each of } P_{1}, P_{2}, \ldots, P_{m} \text { is a consequence of } V^{n}\right\} \text {. }
$$

It follows from Magnus' solution to the word problem for one-relator groups $[12, \S 4.4]$ that there is an algorithm to determine $V$, and that the algorithm is independent of the choice of element of $S$ (see also Corollary 1 of [20]). Now $\left\langle x_{1}, x_{2}, \ldots, x_{r} ; P_{1}, P_{2}, \ldots, P_{m}\right\rangle$ is a presentation of $G$ if and only if there is an element $V_{0}$ of $V$ which is an automorphic image of $R$ or $R^{-1}$ (by Lemma 1), and the existence of the algorithm required in part (ii) follows using Theorem N2 of [12]. This completes the proof of Theorem 1.

Part (i) of Theorem 1 is obviously of importance, in relation to Problem 19 of [27] (see also the first problem mentioned in [2]). It is worth commenting that there are groups with more than one $T$-system which satisfy the assumptions of Theorem 1(i). An example is provided by the group $G=\left\langle x_{1}, x_{2} ; x_{1}^{2}=x_{2}^{3}\right\rangle$, which actually has infinitely many $T$-systems [24], [6]. It should also be pointed out that, contrary to a conjecture of Magnus, not every one-relator group satisfies the assumptions of Theorem 1(i) ([4], [13], and $\S 1.3$ below).

If a one-relator group has one $T$-system then not only can the isomorphism problem be solved in various instances, but other important information can often be deduced.

THEOREM 2. Let $G=\left\langle x_{1}, x_{2}, \ldots, x_{r} ; R^{n}\right\rangle$ where $\left.n\right\rangle$, and suppose $G$ has one T-system. Then $G$ is Hopfian.

This theorem is of importance in view of the conjecture of Baumslag [1] that every one-relator group with torsion is Hopfian.

To prove Theorem 2 it can be assumed that $R$ is nonempty, for otherwise the result is well-known [12, Theorem 2.13]. Moreover, it is no loss of generality to assume that $R$ is not a true power.

Suppose that $N$ is a normal subgroup of $F_{r}$ such that $F_{r} / N$ is isomorphic to $G$. Since $G$ has one $T$-system there is an automorphism $\psi$ of $F_{r}$ such that $N$ is generated as a normal subgroup by $\psi\left(R^{n}\right)$. To prove the theorem, therefore, it suffices to show that if $\psi_{1}$ and $\psi_{2}$ are automorphisms of $F_{r}$ and $\psi_{1}\left(R^{n}\right)$ is a consequence of $\psi_{2}\left(R^{n}\right)$, then $\psi_{1}(R)$ is a conjugate of $\psi_{2}(R)$ or its inverse. Let $\psi_{1}(R)=U$ and $\psi_{2}(R)=V$. Clearly it can be assumed that $V$ is cyclically reduced, and that no automorphic image of $U$ has length less than $U$ (so in particular $L(U) \leqslant L(V)$ ).

Consider first the case $n>2$. If $U^{n}$ is a consequence of $V^{n}$ then by Newman's Spelling Theorem, there are cyclic permutations $S$ and $T$ of $U$ and $V^{\epsilon}$ respectively such that $S^{n} \equiv T^{n-1} T_{1} X$ where $T \equiv T_{1} T_{2}$ and $T_{1}$ is nonempty. Thus 
$T_{2}^{-1} X$ is a consequence of $V^{n}$. Since $L\left(T_{2}\right)+L(X)<(n-1) L(V)$, it follows from Newman's Spelling Theorem that $T_{2}^{-1} X$ is freely equal to the empty word, and so $U$ is a cyclic permutation of $V$ or $V^{-1}$ as required.

Now assume $n=2$, and suppose $U^{2}$ is a consequence of $V^{2}$. Then Newman's Spelling Theorem implies that there are cyclic permutations $S$ and $T$ of $U$ and $V^{\epsilon}$ respectively, such that $T$ is an initial segment of $S^{2}$. Consequently $T \equiv$ $T^{\prime} \bar{U} T^{\prime}$ and $S \equiv T^{\prime} \bar{U}$, and $\bar{U}$ cannot be empty since it was assumed that $R$ is not a true power. Now $\bar{U}^{2}$ is a consequence of $\left(T^{\prime} \bar{U} T^{\prime}\right)^{2}$ and $\left(T^{\prime} \bar{U}\right)^{2}$, and so $T^{\prime}$ must be empty. For otherwise the above argument could be repeated with $\bar{U}$ in place of $U$ to obtain a word $\overline{\bar{U}}$ where $0<L(\overline{\bar{U}})<L(\bar{U})$ and $\overline{\bar{U}}^{2}$ is a consequence of $V^{2}$. And then the argument could be repeated with $\overline{\bar{U}}$ in place of $\bar{U}$, and so on. Obviously this leads to a contradiction, for there are only finitely many words having length less than that of $U$. The proof of the theorem is now complete.

As a corollary of the proof of Theorem 2, the following result is obtained.

Let $F$ be a finitely generated free group and let $N$ be the smallest normal subgroup of $F$ containing the nth power, $n>1$, of an element of $F$. Then $N$ cannot be mapped onto a proper supergroup by an automorphism of $F$.

This result should be compared with Theorem 22.1 of [17].

Theorem 2, when used in conjunction with results obtained in $\S \S 1.3,1.4$ below (see also Theorem 1 of [22]), establishes the Hopficity of groups in a class larger than that considered by Baumslag in [1].

There are several questions of interest concerning $T$-systems of groups in general, and of one-relator groups in particular. Probably the most important of these (in view of Theorems 1 and 2) is the problem of showing that every (finitely generated) one-relator group $G$ with torsion has one $T$-system. In fact, it might reasonably be expected that, except when the relator of $G$ is a power of a primitive, $G$ has one Nielsen equivalence class.

A less ambitious question is prompted by the following considerations. Let $G=\left\langle\dot{x}_{1}, \mathrm{x}_{2}, \ldots, x_{r} ; R^{n}\right\rangle$ and let $\pi(G)$ denote the subgroup of $G$ generated by the elements of finite order. Then it sometimes happens that a property which holds for $G / \pi(G)$ also holds for $G$. (See, for example, Theorem 3 of [1] and Corollary 4.13.1 of [12].) One is led to ask, therefore, whether the fact that $G / \pi(G)$ has one Nielsen equivalence class ( $T$-system) implies a similar conclusion for $G$ itself.

A third question, due to Rosenberger [25], concerns automorphisms. It is not difficult to show that if the $T$-system and Nielsen equivalence class containing a generating $r$-tuple $g$ of a group $G$ coincide, then every automorphism of $G$ can be represented as a free automorphism acting on $\mathbf{g}$. (The converse is also true.) Consequently there is a homomorphism of a subgroup $B$ of the automorphism 
group $A$ of $F_{r}$ onto the automorphism group of $G$. Now in general $G$ may have more than one Nielsen equivalence class (or what amounts here to the same thing, more than one $T$-system), but can this be the case if $B=A$ ? Rosenberger [25] has shown the answer to be "no" if $G$ is a one-relator group.

1.2 Nielsen equivalence classes of certain HNN groups. The previous subsection shows that a determination of the Nielsen equivalence classes and $T$-systems of a group, particularly a one-relator group, is of considerable importance, and this problem has received the attention of several authors (see, for example, $[4]-[6],[13],[16],[18],[19],[22]-[26])$. In this subsection a theorem is obtained which will be used in $\S 1.3$ below to show that certain two-generator onerelator groups with torsion have one Nielsen equivalence class. The theorem is also applicable to some torsion free one-relator groups, as well as to certain twogenerator groups with more than one relator.

It is now well established that a fruitful approach to one-relator groups is to use the fact that they are $H N N$ groups, and this viewpoint will be adopted here. It is therefore convenient to list some of the information about $H N N$ groups which will be needed in the sequel.

Let $G=\left\langle a, b, c, \ldots, t ; P, Q, R, \ldots, t^{-1} U_{j} t=V_{j}(j \in J)\right\rangle$ where $P, Q$, $R, \ldots, U_{j}, V_{j}(j \in J)$ are words in $a, b, c, \ldots$, and let $H=\langle a, b, c, \ldots$; $P, Q, R, \ldots\rangle$. Then $G$ is called an $H N N$ group with base $H$ and stable letter $t$ if the mapping $U_{j} \rightarrow V_{j}, j \in J$, defines an isomorphism $\theta$ of the subgroup $K_{-1}$ of $H$ generated by the $U_{j}$ onto the subgroup $K_{1}$ of $H$ generated by the $V_{j}$. It is usual to call $K_{-1}$ and $K_{1}$ the associated subgroups of $G$. A theorem of Higman, Neumann and Neumann [9] asserts that if $G$ is an $H N N$ group then $H$ is embedded in $G$ by the mapping $a \rightarrow a, b \rightarrow b, c \rightarrow c, \ldots$, and so $H$ can be regarded as a subgroup of $G$ in the natural way. This will be done in the sequel.

Let $W$ be a word in the generators $a, b, c, \ldots, t$ of $G$. The $t$-length of $W$ is the number of $t$-symbols appearing in $W$; this number is denoted by $|W|$. If $|W|=0$ then $W$ is said to be $t$-free. The $t$-length of a pair $\left(W_{1}, W_{2}\right)$ of words is the sum $\left|W_{1}\right|+\left|W_{2}\right|$ and is denoted by $\left|\left(W_{1}, W_{2}\right)\right|$.

A word in the generators $a, b, c, \ldots, t$ of $G$ is said to be $t$-reduced if and only if it has no subword of the form $t^{-\epsilon} k t^{\epsilon}$ where $k$ is $t$-free and belongs to $K_{-\epsilon}$. The importance of $t$-reduced words stems from the following result.

BRITTON'S Lemma. Let $G$ be an HNN group with stable letter $t$. A t-reduced word in the generators of $G$ which involves $t$ is not equal in $G$ to a $t$-free word.

For a proof see [3] or [14].

Given a word $W$ in the generators of $G$ it is reasonably obvious that by 
repeating finitely often the operation of replacing a subword of the form $t^{-\epsilon} k t^{\epsilon}$ by $\theta^{\epsilon}(k)$, a word $U$ can be obtained from $W$, with $|U| \leqslant|W|$, such that $U$ is $t$-reduced and $U=W$ in $G$. Since one of the concerns of this paper is with decidability questions, a specific procedure for obtaining a $t$-reduced word from $W$ will be given. This procedure is algorithmic provided the following two conditions are satisfed.

(i) The set $J$ indexing the generators of $K_{-1}$ and $K_{1}$ is finite.

(ii) The generalized word problem is solvable for $K_{-1}$ and $K_{1}$ in $H$; that is, there is an algorithm to decide for any word $h$ in $a, b, c, \ldots$ whether or not $h$ is equal in $H$ to a word in the generators $U_{j}\left(V_{j}\right)$ of $K_{-1}\left(K_{1}\right)$ and if so for writing $h$ in at least one way in terms of the generators.

It should be mentioned that it can and always will be assumed, regardless of whether the rewriting of elements of $K_{\epsilon}$ in terms of the generators of $K_{\epsilon}$ is algorithmic or not, that if $h$ is a word in $a, b, c, \ldots$ which is freely equal to the empty word then $h$ is rewritten as the empty word in terms of the generators of $K_{\epsilon}$. (This is to ensure that words such as $t^{-1} a^{-1}$ at $t$-reduce to the empty word.)

Now suppose $W$ is a word in $a, b, c, \ldots, t$. Scan $W$ from left to right searching for a subword of the form $t^{-\epsilon} k t^{\epsilon}$ where $k$ is $t$-free and belongs to $K_{-\epsilon}$. If no such subword exists then stop. Otherwise choose the leftmost subword $t^{-\epsilon} k t^{\epsilon}$ of $W$. Write $k$ in terms of the generators of $K_{-\epsilon}$ and apply the isomorphism $\theta^{\epsilon}$ to get a word in the generators of $K_{\epsilon}$. Regard this as a word $k^{*}$ in the generators $a, b, c, \ldots$ of $H$ and replace the subword $t^{-\epsilon} k t^{\epsilon}$ by $k^{*}$. Let $W^{\prime}$ be the resulting word. Then $W^{\prime}=W$ in $G$ and $\left|W^{\prime}\right|<|W|$. Now repeat the above procedure with $W^{\prime}$ in place of $W$, and so on. After a finite number of steps a word $U$ will be reached which is $t$-reduced and is equal to $W$ in $G$. The procedure just described will be called $t$-reducing and $U$ will be called the $t$-reduced form of $W$.

A word in the generators of $G$ is said to be cyclically t-reduced if all its cyclic permutations are $t$-reduced. Thus if $W$ is cyclically $t$-reduced then no conjugate of $W$ is equal in $G$ to a word having $t$-length less than that of $W$. It therefore follows from Britton's Lemma that a cyclically t-reduced word which involves $t$ is not equal in $G$ to a conjugate of an element of $H$.

Given any word $W$ in the generators $a, b, c, \ldots, t$ of $G$ there is a method for obtaining from $W$ a cyclically $t$-reduced word $V$ which is equal in $G$ to a conjugate of $W$ (or, as will be said, is conjugate to $W$ in $G$ ). This method is algorithmic if $t$-reducing is algorithmic. The method is as follows. First $t$-reduce $W$ to obtain $U$. Check whether or not $U U$ is $t$-reduced. If it is then stop. Otherwise let $S$ be the initial segment of $U$ up to and including the first $t$-symbol, and $t$-reduce $S^{-1} U S$ to obtain a word $U^{\prime}$ conjugate to $W$ in $G$ with $\left|U^{\prime}\right|<|U|$. Now repeat 
the procedure with $U^{\prime}$ in place of $U$, and so on. After a finite number of steps a word $V$ will be reached which is cyclically $t$-reduced and conjugate to $W$ in $G$. The word $V$ will be called the cyclically t-reduced form of $W$.

A discussion of the possible Nielsen equivalence classes of two-generator $H N N$ groups will now be given. Precise statements and detailed proof of the results mentioned in the following two paragraphs will be found in $\S 2$.

Suppose $G$ is a two-generator $H N N$ group with base $H$, stable letter $t$, and associated subgroups $K_{-1}$ and $K_{1}$. It is easy to show that any generating pair of $G$ is Nielsen equivalent to a pair of the form $(t X, Y)$ where $X$ and $Y$ belong to the normal subgroup of $G$ generated by $H$. Of more significance for applications, however, is the fact that under appropriate conditions $X$ and $Y$ may be assumed to be elements of $H$ itself. In fact $Y$ may be assumed to belong to $H$ without imposing any further restrictions on $G$. (See the First Reduction Theorem in §2.2.) On the other hand, to deduce that $X$ can also be taken as an element of $H$, it is found necessary to suppose that $K_{-1}$ and $K_{1}$ are malnormal in $H$. (See the Second Reduction Theorem in $\S 2.3$. The reader is reminded that a subgroup $A$ of a group $B$ is malnormal in $B$ if for all elements $b$ of $B, b^{-1} A b \cap A \neq 1$ implies $b \in A$.) The conclusion that $X$ and $Y$ may be taken as elements of $H$ is not in actual fact the best possible. With very little extra effort it can be shown that $Y$ may actually be assumed to belong to $K_{-1}$.

Even more is true. Suppose that in addition to the assumption that $K_{-1}$ and $K_{1}$ are malnormal in $H$ it is assumed that the generalized word problem is solvable for $K_{-1}$ and $K_{1}$ in $H$. Then there is an algorithmic procedure which when applied to any pair of words $\left(W_{1}, W_{2}\right)$ in the generators of $G$ enables one either to decide that $\operatorname{sgp}\left\{W_{1}, W_{2}\right\} \neq G$, or to produce a pair $(t h, k)$ with $h \in H$ and $k \in K_{-1}$, where the pair $(t h, k)$ is Nielsen equivalent to $\left(W_{1}, W_{2}\right)$ if $\operatorname{sgp}\left\{W_{1}, W_{2}\right\}=G$. (See $\S \S 2.2,2.3$.)

The general results outlined above are applicable to two-generator one-relator groups with torsion in which the relator has zero sum exponent on one of the generators. Suppose $G=\left\langle a, t ; R^{n}\right\rangle$ where $R$ is a cyclically reduced word involving $a, t$ and where the exponent sum of $R$ on $t$ is zero, and let $a_{i} \quad(i=0, \pm 1$, $\pm 2, \ldots)$ denote the word $t^{-i} a t^{i}$. Then, as observed by Moldavanskii [15] , if $P$ is the word obtained from $R$ by rewriting it in terms of the $a_{i}$, and if $m$ and $M$ are, respectively, the least and greatest integers $i$ for which $a_{i}$ occurs in $P$ then $G$ can be presented as an $H N N$ group as follows:

$$
G=\left\langle a_{m}, \ldots, a_{M}, t ; P^{n}, t^{-1} a_{i} t=a_{i+1} \quad(i=m, \ldots, M-1)\right\rangle .
$$

Now the associated subgroups $\operatorname{sgp}\left\{a_{m}, \ldots, a_{M-1}\right\}$ and $\operatorname{sgp}\left\{a_{m+1}, \ldots, a_{M}\right\}$ are malnormal in the base $H=\left\langle a_{m}, \ldots, a_{M} ; P^{n}\right\rangle$ (see [20]), and the generalized 
word problem for $H$ relative to the associated subgroups is solvable [12, Theorem 4.14].

The situation considered here is when $M-m=1$. More generally, interest will centre on noncyclic $H N N$ groups of the form

$$
G=\left\langle a, b, t ; R_{1}, R_{2}, \ldots, t^{-1} c t=d\right\rangle,
$$

where $R_{1}, R_{2}, \ldots, c, d$ are words in $a, b$ and $(c, d)$ is a generating pair of the group $H=\left\langle a, b ; R_{1}, R_{2}, \ldots\right\rangle$. It follows from previous discussion that if sgp $\{c\}$ and sgp $\{d\}$ are malnormal in $H$ then any generating pair of $G$ is Nielsen equivalent to a pair of the form $\left(t h, c^{\mu}\right)$ where $h \in H$. Moreover, if the generalized word problem is solvable for sgp $\{c\}$ and $\operatorname{sgp}\{d\}$ in $H$ then there is an algorithm to decide for any pair of words $\left(W_{1}, W_{2}\right)$ in $a, b, t$ whether or not $\operatorname{sgp}\left\{W_{1}, W_{2}\right\}=G$, provided there is an algorithm to decide which pairs of the form $\left(t h, c^{\mu}\right)(h \in H)$ generate $G$.

Now by slightly modifying the proof of Lemma 1 of [22] it can be shown that $\left(t h, c^{\mu}\right)$ generates the group $G$ of the previous paragraph only if $c$ and $h^{-1} d h$ generate $H$. In many instances $c$ and $h^{-1} d h$ will generate $H$ if and only if $h$ is expressible in the form $d^{\delta} c^{\gamma}$. When this is the case it follows that any generating pair of $G$ is Nielsen equivalent to a pair of the form $\left(c^{\delta} t c^{\gamma}, c^{\mu}\right)$. The question then arises as to which pairs of this form can generate $G$. To answer this question it suffices to assume that $\gamma=\delta=0$. For the mapping $t \rightarrow c^{\delta} t c^{\gamma}, c \rightarrow c$, $d \rightarrow c^{-\gamma} d c^{\gamma}$ is easily seen to define an automorphism of $G$.

Suppose that for every integer $\lambda$,

$$
\operatorname{sgp}\left\{c^{\lambda}, d^{\lambda}\right\} \cap \operatorname{sgp}\{c\}=\operatorname{sgp}\left\{c^{\lambda}\right\}
$$

and

$$
\operatorname{sgp}\left\{c^{\lambda}, d^{\lambda}\right\} \cap \operatorname{sgp}\{d\}=\operatorname{sgp}\left\{d^{\lambda}\right\}
$$

and consider a pair $\left(t, c^{\mu}\right)$. Let $W$ be a word in $t, c^{\mu}$ and $d^{\mu} \quad\left(=t^{-1} c^{\mu} t\right)$. Then it is not difficult to see that $W$ is equal in $G$ to a $t$-reduced word of the form $h_{0} t^{\epsilon} h_{1} t^{\epsilon}{ }^{\epsilon} h_{2} \cdots t^{\epsilon} h_{r}$ where the $h_{i}$ are words in $c^{\mu}$ and $d^{\mu}$. This is proved by induction on the number of $t$-symbols in $W$. If this number is zero then the result is obvious. Assume the result holds for all words with less than $s t$-symbols, where $s>0$, and suppose $W \equiv w_{0} t^{\delta}{ }_{1} w_{1} t^{\delta}{ }^{\delta} w_{2} \cdots t^{\delta} s_{w_{s}}$. Here the $w_{i}$ are words in $c^{\mu}$ and $d^{\mu}$ and $\left|\delta_{i}\right|=1$ for $i=1,2, \ldots, s$. If $W$ is $t$-reduced the result holds. Otherwise $W$ has a subword of the form $t^{-\epsilon} k t^{\epsilon}$ where $k$ is $t$-free and belongs to $K_{-\epsilon}$. Therefore, by (1.1) and (1.2), either $k$ is equal in $H$ to a power, $c^{m \mu}$, of $c^{\mu}$ (if $\epsilon=1$ ), or $k$ is equal in $H$ to a power, $d^{n \mu}$, of $d^{\mu}$ (if $\epsilon=-1$ ). In the former case replace $t^{-\epsilon} k t^{\epsilon}$ by $d^{m \mu}$, and in the latter by $c^{n \mu}$. Then in either case a word $W^{*}$ in 
$t, c^{\mu}, d^{\mu}$ is obtained which is equal to $W$ in $G$ and has less $t$-symbols. The inductive hypothesis then gives the desired result.

Now it follows from what has first been shown, together with Britton's Lemma, that

$$
\operatorname{sgp}\left\{c^{\mu}, t\right\} \cap H=\operatorname{sgp}\left\{c^{\mu}, d^{\mu}\right\} .
$$

Therefore (1.1) implies that $t$ and $c^{\mu}$ generate $G$ if and only if $\mu$ is coprime to the order of $c$; for otherwise $c \notin \operatorname{sgp}\left\{c^{\mu}, t\right\}$.

It is important to observe that if it is assumed in the previous three paragraphs that the order of $c$ is known and that there is an algorithm to decide for any word $h$ in $a, b$ whether or not $h$ is expressible in the form $d^{\delta} c^{\gamma}$, then it can be effectively decided whether or not a pair of the form $\left(t h, c^{\mu}\right)$ generates $G$.

The above discussion motivates the introduction of the following classes of groups. Let $H$ be a two-generator group, and let $(c, d)$ be a generating pair of $H$. Then $H$ will be called an $F E\left({ }^{2}\right)$ group relative to $(c, d)$ (or simply an $\mathrm{FE}$ group if $c$ and $d$ are understood or if it is immaterial what $c$ and $d$ are) if the following conditions are satisfied.

Condition 1. $c$ and $d$ have equal order.

Condition 2. For every integer $\lambda, \operatorname{sgp}\left\{c^{\lambda}, d^{\lambda}\right\} \cap \operatorname{sgp}\{c\}=\operatorname{sgp}\left\{c^{\lambda}\right\}$ and $\operatorname{sgp}\left\{c^{\lambda}, d^{\lambda}\right\} \cap \operatorname{sgp}\{d\}=\operatorname{sgp}\left\{d^{\lambda}\right\}$.

Condition 3. $\operatorname{sgp}\{c\}$ and sgp $\{d\}$ are malnormal in $H$.

Condition 4. If $c$ and $h^{-1} d h$ generate $H$ then $h$ is expressible in the form $d^{\delta} c^{\lambda}$.

Now suppose $H=\left\langle a, b ; R_{1}, R_{2}, \ldots\right\rangle$, and suppose $c, d$ are given as words in $a, b$. Then $H$ will be called an $A F E\left({ }^{3}\right)$ group (relative to $(c, d)$ ) if it is an FE group and in addition the following hold.

Condition 5. The order of $c$ is known.

CONDITION 6. There is an algorithm to determine for any word in $a, b$ whether or not it is expressible in the form $d^{\delta} c^{\lambda}$.

Condition 7. The generalized word problem is solvable for sgp $\{c\}$ and sgp $\{d\}$ in $H$.

Using these definitions, the above results, when combined with the general theory discussed previously, lead to the following theorem.

THEOREM 3. Let $H=\left\langle a, b ; R_{1}, R_{2}, \ldots\right\rangle$ and suppose $H$ is a nontrivial $F E$ group relative to $(c, d)$. Let $G=\left\langle a, b, t ; R_{1}, R_{2}, \ldots, t^{-1} c t=d\right\rangle$. Then any generating pair of $G$ is Nielsen equivalent to a pair of the form $\left(t, c^{\mu}\right)$ where

(2) Free enough.

(3) The "A" stands for algorithm. 
$\mu$ is coprime to the order of $c$. If $H$ is an AFE group then the two-generator problem is solvable for $G$.

(The two-generator problem (2GP) for a group $G$ given by a presentation $\left\langle b_{1}, b_{2}, \ldots, b_{p} ; P_{1}, P_{2}, \ldots\right\rangle$ is the algorithmic problem of deciding for any pair of words $\left(W_{1}, W_{2}\right)$ in $b_{1}, b_{2}, \ldots, b_{p}$ whether or not $W_{1}$ and $W_{2}$ generate $G$.)

1.3 Applications of Theorem 3. In order to apply Theorem 3 it is of course necessary to carry out a determination of FE and AFE groups. When looking for groups which satisfy some or all of Conditions 1-7 it is reasonable to consider those groups which are known to be combinatorially similar to $F_{2}$. Three such classes of groups are one-relator groups with torsion, small cancellation groups and Fuchsian groups. (These classes are not of course disjoint.) Results are obtained in $\S 3$ for each of these three types of groups. The results are listed here.

In the following, a certain presentation on $a, b$ of a group $H$ is given. After this is given a pair of words $(c, d)$ in $a, b$ which generate $H$. Then it is indicated whether $H$ is an FE or AFE group relative to $(c, d)$.

(1.3) $\left.\left\langle a, b ; P^{n}\right\rangle, n\right\rangle 1, P$ a cyclically reduced word involving $a$ and $b$, all the exponents to which a occurs in $P$ have the same sign, if $n=2$ then all the exponents to which $b$ occurs in $P$ have the same sign; $(a, b) ; A F E$.

Let $W$ be a word in two variables $y_{-1}$ and $y_{1}$. Then nonzero integers $\xi, \eta$ will be called adjacent exponents in $W$ if $W \equiv U y_{\epsilon}^{\xi} y_{-\epsilon}^{\eta} V$, where $U$ is either empty or has last symbol $y_{-\epsilon}$ or $y_{-\epsilon}^{-1}$, and $V$ is either empty or has first symbol either $y_{\epsilon}$ or $y_{\epsilon}^{-1}$.

(1.4) $\left\langle a, b ; R_{1}, R_{2}, \ldots\right\rangle, R_{1}, R_{2}, \ldots$ are cyclically reduced, the presentation satisfies the small cancellation condition $C^{\prime}(1 / 18)[11]$, in each relator either all the exponents to which a occurs have the same sign or all the exponents to which $b$ occurs have the same sign, $a^{\kappa}$ is a relator if and only if $b^{\kappa}$ is, adjacent exponents in each relator are coprime; $\left(a^{\xi}, b\right)$ where $\xi$ is coprime to the order of $a ; F E, A F E$ if finitely presented.

If one supposes that each relator is either a positive or negative word then $C^{\prime}(1 / 18)$ can be replaced by $C^{\prime}(1 / 8)$ in the above.

(1.5) $\left\langle a, b ; a^{p}, b^{p},(a b)^{q}\right\rangle, 2 / p+1 / q<1 ;\left(a^{\xi}, b\right)$ where $\xi$ is coprime to $p$; $F E, A F E$ if $p \geqslant 4$.

As well as the triangle groups in (1.5) the Fuchsian groups with presentation of the form $\left.\left\langle a, b ;[a, b]^{n}\right\rangle(n\rangle 1\right)$ are AFE groups. This follows from results obtained in $\$ 1.4$.

By using the groups listed above several interesting results can be deduced from Theorem 3. For example, using (1.3) the following can easily be verified. If $G=\left\langle a, t ;\left(a^{\alpha_{1}} t^{-1} a^{\beta_{1}} t \cdots a^{\alpha_{s}} t^{-1} a^{\beta_{s}} t\right)^{n}\right\rangle$ where $\left.n\right\rangle 1$, the $\alpha_{i}$ are non- 
zero and have the same sign, the $\beta_{i}$ are nonzero, if $n=2$ the $\beta_{i}$ have the same sign, then $G$ has one Nielsen equivalence class. Moreover, 2GP is solvable for $G$.

This generalizes Theorem 1 of [22]. It should be mentioned that the restriction $n>1$ cannot be removed in general in the first part of the theorem, even if it is only required that $G$ have one $T$-system. For Brunner [4] has shown that if $B=\left\langle a, t ; a^{3} t^{-1} a^{-2} t\right\rangle$ then $B$ has infinitely many $T$-systems.

A proof that the group $B$ with presentation $\left\langle a, t ; a^{3} t^{-1} a^{-2} t\right\rangle$ has two generating pairs lying in different $T$-systems, each associated with a one-relator presentation of $B$, can be given as follows. Note that $(a, t)$ and $\left(a^{2}, t\right)$ both generate $B$, and so $B$ has the two presentations $\left\langle x_{1}, x_{2} ; x_{1}^{3} x_{2}^{-1} x_{1}^{-2} x_{2}\right\rangle,\left\langle x_{1}, x_{2}\right.$; $\left.x_{1}\left[x_{1}^{-1}, x_{2}\right]^{2}\right\rangle$. Let $Q_{1} \equiv x_{1}^{3} x_{2}^{-1} x_{1}^{-2} x_{2}$ and $Q_{2} \equiv x_{1}\left[x_{1}^{-1}, x_{2}\right]^{2}$. If $(a, t)$ and $\left(a^{2}, t\right)$ lay in the same $T$-system then there would be an automorphism $\psi$ of $F_{2}$ mapping $Q_{1}$ to $Q_{2}$ or $Q_{2}^{-1}$, by Lemma 1 . Now necessarily, the exponent sum of $\psi\left(x_{1}\right)$ on $x_{2}$ would have to be zero, so that $\psi$ would differ by an inner automorphism from an automorphism of the form $x_{1} \rightarrow x_{1}^{\epsilon}, x_{2} \rightarrow x_{1}^{\lambda} x_{2}^{\epsilon^{\prime}}$ [12, Corollary N4]. However, no such automorphism can map $Q_{1}$ to $Q_{2}^{ \pm 1}$, and so $(a, t)$ and $\left(a^{2}, t\right)$ do not lie in the same $T$-system. It should be mentioned that the fact that $\left\langle x_{1}, x_{2} ; Q_{1}\right\rangle$ and $\left\langle x_{1}, x_{2} ; Q_{2}\right\rangle$ are presentations of $B$ associated with generating pairs from different $T$-systems, when used in conjunction with the theorem stated above, shows the existence of two words $U$ and $V$ in $x_{1}, x_{2}$ such that groups with presentations $\left\langle x_{1}, x_{2} ; U^{n}\right\rangle,\left\langle x_{1}, x_{2} ; V^{n}\right\rangle(n>0)$ are isomorphic if and only if $n=1$. (See Corollary 3 of [22].)

An argument similar to that in the previous paragraph can be used to establish the following result of $\mathrm{McCool}$ and Pietrowski [13]. Given a positive integer $m$ there are words $Q_{1}, Q_{2}, \ldots, Q_{m}$ in $x_{1}, x_{2}$ such that groups with presentations $\left\langle x_{1}, x_{2} ; Q_{i}\right\rangle(i=1,2, \ldots, m)$ are isomorphic, but there is no automorphism of $F_{2}$ mapping $Q_{i}$ to $Q_{j}^{ \pm 1}$ for $i \neq j$. For example, let $Q_{i} \equiv$ $x_{1}\left[x_{1}^{-2^{m-i}}, x_{2}\right]^{2}$. Then $\left\langle x_{1}, x_{2} ; Q_{i}\right\rangle$ is a presentation of the group $G=\langle a, t$; $\left.a^{2^{m}+1} t^{-1} a^{-2^{m}} t\right\rangle$. The question (raised by $\mathrm{McCool}$ and Pietrowski) remains open as to whether the restriction that $m$ be finite can be removed in the result just quoted.

In contradistinction to the results of the previous two paragraphs there is a large number of words $R$ of the form

$$
a^{\alpha_{1}} t^{-1} a^{\beta_{1}} t \cdots a^{\alpha_{s}} t^{-1} a^{\beta} s_{t}
$$

$\left(s>0, \alpha_{i}>0\right.$ for $i=1,2, \ldots, s, \beta_{i} \neq 0$ for $\left.i=1,2, \ldots, s\right)$ which are not proper powers, for which a group with presentation $\langle a, t ; R\rangle$ has one Nielsen equivalence class. This can be deduced using (1.4) together with Theorem 3. For example, the following result can easily be proved. 
If $R$ has the form (1.6) and the $\alpha_{i}$ are pairwise distinct, the $\beta_{i}$ are pairwise distinct, adjacent integers in the $2 s$-tuple $\left(\alpha_{1}, \beta_{1}, \ldots, \alpha_{s}, \beta_{s}\right)$ are coprime,

$$
\frac{\max \left\{\alpha_{i}+\left|\beta_{i}\right|: i=1,2, \ldots, s\right\}}{\sum_{i=1}^{s}\left(\alpha_{i}+\left|\beta_{i}\right|\right)}<\frac{1}{18},
$$

then a group with presentation $\langle a, t ; R\rangle$ has one Nielsen equivalence class. Moreover, $2 \mathrm{GP}$ is solvable for $G$.

Of course, information about the Nielsen equivalence classes, and a solution to the two-generator problem, for other groups, including many with more than one relator, can also be obtained by using (1.4) together with Theorem 3 .

The following result can be derived easily from (1.5) and Theorem 3 . If $G=\left\langle a, t ; a^{p},\left(a t^{-1} a^{\xi} t\right)^{q}\right\rangle$ where $2 / p+1 / q<1$ and $\xi$ is coprime to $p$, then any generating pair of $G$ is Nielsen equivalent to a pair of the form $\left(a^{\mu}, t\right)$, where $\mu$ is coprime to $p$. Also if $p \geqslant 4$ then $2 \mathrm{GP}$ is solvable for $G$.

1.4 Construction of FE groups. A natural question to ask is how one may construct FE groups. A method of doing this is given here. Let $H$ be a two-generator group generated by $c$ and $d$. Then $H$ will be called special (relative to $(c, d)$ ) if the following holds.

Condition 8. No nontrivial power of $c$ is conjugate to a power of $d$. The groups listed in (1.3) and (1.4) are special.

THEOREM 4. Let $H=\left\langle a, b ; R_{1}, R_{2} \cdots\right\rangle$ and suppose a has infinite order, and that $H$ is a special $F E$ group relative to $(a, b)$. Let $G=\left\langle a, b, t, R_{1}, R_{2}\right.$, $\left.\ldots, t^{-1} a^{\epsilon} t=b\right\rangle$. Then $G$ is a special FE group relative to $(a, t)$. Moreover, if $H$ is a special AFE group relative to $(a, b)$, then $G$ (when presented on $a, t)$ is $a$ special $A F E$ group relative to $(a, t)$.

This theorem, when combined with Theorem 3, gives an iterative procedure for showing that certain groups have one Nielsen equivalence class. For example, let $H_{0}=\left\langle a, b_{0} ;\left(a^{-\epsilon_{1}} b_{0}\right)^{n}\right\rangle$ where $\left.n\right\rangle 1$. Then $H_{0}$ is a special AFE group relative to $\left(a, b_{0}\right)$ (see (1.3)) and $a$ has infinite order by the Freiheitssatz. It therefore follows from Theorem 4 that if $H_{1}=\left\langle a, b_{1} ;\left[a^{\epsilon}, b_{1}\right]^{n}\right\rangle$ then $H_{1}$ is a special AFE group relative to $\left(a, b_{1}\right)$. Moreover, $H_{1}$ has one Nielsen equivalence class, and has solvable two-generator problem, by Theorem 3. Now if $\mathrm{H}_{2}=$ $\left\langle a, b_{2} ;\left[a^{\epsilon_{1}},\left[a^{\epsilon_{2}}, b_{2}\right]\right]^{n}\right\rangle$, then again by Theorem $4, H_{2}$ is a special AFE group relative to $\left(a, b_{2}\right)$; and $\mathrm{H}_{2}$ has one Nielsen equivalence class and solvable twogenerator problem by Theorem 3. And so on. Finally, after $r$ steps $(r>0)$ it is concluded that if $G=\left\langle a, t ;\left[x^{\epsilon_{1}},\left[a^{\epsilon_{2}},\left[\ldots,\left[a^{\epsilon_{r}}, t\right] \ldots\right]\right]\right]^{n}\right\rangle$, then $G$ has one Nielsen equivalence class, and $\mathbf{2 G P}$ is solvable for $G$. 
Let $E_{r}(r \geqslant 0)$ denote a word of the form $\left[a^{\epsilon_{1}},\left[a^{\epsilon_{2}},\left[\ldots,\left[a^{\epsilon_{r}}, t\right] \ldots\right]\right]\right]$. Then the above argument can obviously be generalized to establish the following theorem.

Let $H=\left\langle a, b ; a^{\alpha_{1}} b^{\beta_{1}} \cdots a^{\alpha_{s}} b^{\beta_{s}}\right\rangle$ where the $\alpha_{i}$ and $\beta_{i}$ are nonzero integers, and suppose $H$ is a special $F E$ group relative to $(a, b)$. Let $G=\left\langle a, t ; a^{\alpha}{ }^{1_{1}} E_{r}^{-1} a^{\beta_{1}} E_{r}\right.$ $\left.\cdots a^{\alpha} E_{r}^{-1} a^{\beta} E_{r}\right\rangle$. Then $G$ has one Nielsen equivalence class. If $H$ is a special AFE group then $2 \mathrm{GP}$ is solvable for $G$.

The restriction in this theorem that $H$ be a one-relator group can clearly be dispensed with, provided it is assumed that $a$ has infinite order in $H$. The reader should have no difficulty in formulating a more general result if he wishes to do so.

The above theorem can obviously be used in conjunction with (1.3) and (1.4) to generalize results obtained in $\$ 1.3$.

The rest of this subsection is devoted to proving Theorem 4. Two preparatory lemmas are required. The first of these is needed only briefly in the proof of Theorem 4, but will be used more extensively in $\S 2$. The second lemma is required only for the proof of Theorem 4.

LEMMA 2. Let $G$ be an $H N N$ group with base $H$, stable letter $t$, and associated subgroups $K_{-1}$ and $K_{1}$. Let $X$ and $Y$ be t-reduced words and let $Z$ be the t-reduced form of $X Y$. Suppose that in t-reducing $X Y$ to $Z$ all the $t$-symbols from $X$ are removed. Let $X_{1}$ be an initial segment of $X$. Then $X_{1}^{-1} Z$ is t-reduced. Similarly, if all the t-symbols from $Y$ are removed then $Z Y_{1}^{-1}$. is t-reduced, where $Y_{1}$ is a terminal segment of $Y$.

PRoof. The second part follows from the first by considering $Y^{-1} X^{-1}$. To prove the first part it can be assumed $\left|X_{1}\right|>0$ and $|Z|>0$, for otherwise the result is trivial. Suppose $X_{1}$ has initial segment $x t^{\omega}$ where $x$ is $t$-free and $\omega=$ \pm 1 . Then $Y \equiv Q \bar{Y}$ where $|Q|=|X|, Q$ ends with $t^{-\omega}$ and $X Q t$-reduces to $x k$ where $k$ is $t$-free and belongs to $K_{-\omega}$. Now suppose $\bar{Y}$ has initial segment $y t^{\delta}$ where $y$ is $t$-free and $\delta= \pm 1$. Then $Z$ has initial segment $x k y t^{\delta}$. Now $X_{1}^{-1} Z$ is $t$-reduced unless $\omega=\delta$ and $k y \in K_{-\omega}$. But if $k y \in K_{-\omega}$ then $y \in K_{-\omega}$ and so $\omega \neq \delta$, otherwise $Y$ would not be $t$-reduced. This completes the proof.

It should be remarked that Lemma 2 could also have been proved using the fact (which follows from Britton's Lemma) that if two words are equal in $G$ then their $t$-reduced forms have the same $t$-length.

Lemma 3. Let $G$ and $H$ be as in the statement of Theorem 4. Let $Z$ be a $t$-reduced word involving $t$, and let $a^{\kappa}$ be a nontrivial element of $\operatorname{sgp}\{a\}$. Then $Z^{-1} a^{\kappa} Z$ is equal in $G$ to an element of $H$ if and only if $Z \equiv x t z$ where $x$ and $z$ are $t$-free and $x \in \operatorname{sgp}\{a\}$.

Proof. Sufficiency is obvious. 
To prove necessity, suppose $Z^{-1} a^{\kappa} Z$ defines an element of $H$, and let $Z$ have initial segment $x t^{\omega}$ where $x$ is $t$-free and $|\omega|=1$. Then it follows from Britton's Lemma that either $x^{-1} a^{\kappa} x \in \operatorname{sgp}\{a\}$ (if $\omega=1$ ) or $x^{-1} a^{\kappa} x \in \operatorname{sgp}\{b\}$ (if $\omega=-1$ ). However, the second possibility cannot hold since $H$ satisfies Condition 8. Consequently $\omega=1$ and $x \in \operatorname{sgp}\{a\}$ (by Condition 3). To complete the proof it must be shown that $Z$ cannot involve more than one $t$-symbol. Suppose, by way of contradiction, that $Z$ has initial segment $x t y t^{\delta}$ where $y$ is $t$-free and $|\delta|=1$. Then it follows again from Britton's Lemma that either $y^{-1} b^{\epsilon \kappa} y \in$ $\operatorname{sgp}\{a\}$ (if $\delta=1$ ) or $y^{-1} b^{\epsilon \kappa} y \in \operatorname{sgp}\{b\}$ (if $\delta=-1$ ). However, neither of these possibilities can hold. The former is ruled out because $H$ satisfies Condition 8 , and the latter cannot hold because it would require $y$ to belong to sgp $\{b\}$ (by Condition 3), contradicting the fact that $Z$ is $t$-reduced. This proves the lemma.

In order to prove Theorem 4 it is convenient to introduce a mapping e of $t$-reduced words to integers as follows. Let $Z$ be a $t$-reduced word. It is not difficult to see that one of $t^{-1} Z, t Z$ is $t$-reduced. If $t^{-1} Z$ is $t$-reduced then e( $Z$ ) is defined to be the maximum of the set $A$, where

$$
A=\left\{r: \text { all } t \text {-symbols from } t^{r} \text { are removed in } t \text {-reducing } t^{r} Z\right\} \text {. }
$$

Otherwise e $(Z)$ is defined to be the minimum of $A$.

A proof of Theorem 4 will now be given. First assume that $H$ is a special FE group relative to $(a, b)$. Then it will be shown that $G$ is a special FE group relative to $(a, t)$.

That Condition 1 holds is clear.

Condition 2. Let $\lambda$ be a nonzero integer and let $W$ be a word in $t^{\lambda}$ and $a^{\lambda}$. Suppose that $W$ defines an element of $\operatorname{sgp}\{a\}$. Then since $W$ is a word in $t$, $a^{\lambda}, b^{\lambda}$, and since $H$ satisfies Condition 2, an argument similar to that used in $\S 1.2$ (following (1.2)) shows that $W$ is equal in $G$ to a word in $a^{\lambda}$ and $b^{\lambda}$. Thus $\operatorname{sgp}\left\{a^{\lambda}, t^{\lambda}\right\} \cap H \subseteq \operatorname{sgp}\left\{a^{\lambda}, b^{\lambda}\right\}$, and so $\operatorname{sgp}\left\{a^{\lambda}, t^{\lambda}\right\} \cap \operatorname{sgp}\{a\}=\operatorname{sgp}\left\{a^{\lambda}\right\}$ since $\operatorname{sgp}\left\{a^{\lambda}, b^{\lambda}\right\} \cap \operatorname{sgp}\{a\}=\operatorname{sgp}\left\{a^{\lambda}\right\}$.

Now suppose that $W$ defines an element of $\operatorname{sgp}\{t\}$. By considering the factor group of $G$ by the normal subgroup generated by $H$ it follows easily that $W \in$ $\operatorname{sgp}\left\{t^{\lambda}\right\}$. Thus $\operatorname{sgp}\left\{a^{\lambda}, t^{\lambda}\right\} \cap \operatorname{sgp}\{t\}=\operatorname{sgp}\left\{t^{\lambda}\right\}$.

Condition 3. To show that $\operatorname{sgp}\{a\}$ is malnormal in $G$, first observe that if $g^{-1} a^{\kappa} g \in \operatorname{sgp}\{a\}$, where $\kappa \neq 0$ and $g \in G$, then in fact $g \in H$. For otherwise, by Lemma 2, $g=a^{\alpha}$ th where $h \in H$, and so $g^{-1} a^{\kappa} g=h^{-1} b^{\epsilon \kappa} h$ and $h^{-1} b^{\epsilon \kappa} h \in$ $\operatorname{sgp}\{a\}$. This contradicts the fact that $H$ satisfies Condition 8. Now since $g \in H$ and $\operatorname{sgp}\{a\}$ is malnormal in $H$, it follows that $g \in \operatorname{sgp}\{a\}$ and so $\operatorname{sgp}\{a\}$ is malnormal in $G$.

To prove that $\operatorname{sgp}\{t\}$ is malnormal in $G$, let $W$ be a $t$-reduced word and 
suppose $W^{-1} t^{m} W \in \operatorname{sgp}\{t\}$, where $m \neq 0$. By considering the factor group of $G$ by the normal subgroup generated by $H$ it follows that $W^{-1} t^{m} W=t^{m}$. Let $W_{1}$ be the $t$-reduced form of $t^{\mathrm{e}(W)} W$. Then $W_{1}^{-1} t^{m} W_{1}=t^{m}$ and $W_{1}^{-1} t^{m} W_{1}$ is $t$-reduced by Lemma 2 and the definition of e $(W)$. To complete the proof it suffices to show that $W_{1}=1$ in $G$. Suppose not. Then one of $t^{-m} W_{1}^{-1} t^{m} W_{1}$, $W_{1}^{-1} t^{m} W_{1} t^{-m}$ is a $t$-reduced word involving $t$ and yet defining an element of $H$, contrary to Britton's Lemma. This is obvious if $W_{1}$ involves $t$. On the other hand, if $W_{1}$ does not involve $t$ then the result follows from the fact that $W_{1}$ cannot simultaneously be equal to a power of $a$ and a power of $b$ (by Condition 8).

Condition 4. Let $W$ be a $t$-reduced word and suppose that $a$ and $W^{-1} t W$ generate $G$. Then $\left(a, W^{-1} t W\right)$ is Nielsen equivalent to $(a, t)$, by Theorem 3 , and so it follows from Theorem 3.9 of [12] that $\left[a, W^{-1} t W\right]$ is conjugate in $G$ to $a^{-1} b^{\epsilon} \quad(=[a, t])$. In particular, $\left[a, W^{-1} t W\right]$ is conjugate in $G$ to an element of $H$. It will be shown that this latter statement implies that $W=t^{\tau} a^{\alpha}$ in $G$ for suitable integers $\tau, \alpha$.

Let $W_{1}$ be the $t$-reduced form of $t^{\mathrm{e}(W)} W$. Now it follows from Lemma 1 that either $W_{1} a W_{1}^{-1}$ is $t$-reduced or $W_{1} \equiv W_{2} t^{-1} a^{\lambda}$ and $W_{2} b^{\epsilon} W_{2}^{-1}$ is $t$-reduced. Thus $\left[a, W^{-1} t W\right]$ is conjugate in $G$ to one of $P$ and $Q$ where

$$
P \equiv t^{-1} W_{1} a^{-1} W_{1}^{-1} t W_{1} a W_{1}^{-1}, \quad Q \equiv t^{-1} W_{2} b^{-\epsilon} W_{2}^{-1} t W_{2} b^{\epsilon} W_{2}^{-1} .
$$

Suppose that $\left[a, W^{-1} t W\right]$ is conjugate to $P$. Then the cyclically $t$-reduced form of $P$ has $t$-length zero. Thus $W_{1}$ is $t$-free, for otherwise $P$ is cyclically $t$-reduced by Lemma 2 and the definition of $\mathrm{e}(W)$. Moreover, either $W_{1} a W_{1}^{-1} \in$ $\operatorname{sgp}\{a\}$ or $W_{1} a W_{1}^{-1} \in \operatorname{sgp}\{b\}$. However the second of these possibilities is excluded since $H$ satisfies Condition 8. Thus $W_{1} a W_{1}^{-1} \in \operatorname{sgp}\{a\}$ and so $W_{1} \in$ $\operatorname{sgp}\{a\}$ (since $H$ satisfies Condition 3).

Now suppose that $\left[a, W^{-1} t W\right]$ is conjugate to $Q$. Then an argument similar to that in the previous paragraph establishes that $W_{2}$ is $t$-free and $W_{2} \in \operatorname{sgp}\{b\}$. This however is impossible, for it implies that $t W_{1}$ is not $t$-reduced, which contradicts either Lemma 2 or the definition of $\mathrm{e}(W)$.

Condition 8. Since a nontrivial power of $t$ is cyclically $t$-reduced, it cannot be conjugate in $G$ to an element of $H$ (and in particular to a power of $a$ ). This follows from Britton's Lemma.

Now suppose that $H$ is an AFE group relative to $(a, b)$. Then it is required to show that $G$ satisfies Conditions $5,6,7$ relative to $(a, t)$. That Condition 5 holds is obvious. To see that $G$ satisfies Conditions 6 and 7 first observe that since Condition 7 holds for $H, t$-reducing is algorithmic. For the rest of the discussion $W$ will denote a word in $a, t$ and $U$ will denote the $t$-reduced form of $W$.

Consider first the generalized word problem for $\operatorname{sgp}\{a\}$ in $G$. Note that, by 
Britton's Lemma, $W$ defines an element of sgp $\{a\}$ only if $U$ is $t$-free. Thus the generalized word problem for $\operatorname{sgp}\{a\}$ in $G$ reduces to the generalized word problem for $\operatorname{sgp}\{a\}$ in $H$, and this latter problem is solvable.

Next consider the generalized word problem for $\operatorname{sgp}\{t\}$ in $G$. If $W$ defines an element $t^{\lambda}$ of sgp $\{t\}$, then it follows from Britton's Lemma that the modulus of $\lambda$ is equal to the $t$-length of $U$, and that the $t$-reduced form $V$ of $t^{-\lambda} U$ is $t$-free and defines the identity of $H$. Since the word problem is solvable for $H$, the generalized word problem is solvable for $\operatorname{sgp}\{t\}$ in $G$.

Finally, consider the algorithmic problem of deciding whether or not a word in $a, t$ is expressible in the form $t^{\tau} a^{\alpha}$. Now if $W=t^{\tau} a^{\alpha}$ in $G$ then it follows from Britton's Lemma that the modules of $\tau$ is equal to the $t$-length of $U$, and that the $t$-reduced form of $t^{-\tau} W$ is $t$-free and defines an element of $\operatorname{sgp}\{a\}$. The required algorithm then follows from the fact that the generalized word problem is solvable for sgp $\{a\}$ in $H$.

2. The reduction theorems. The aim of this section is to give precise formulations of the two reduction theorems stated informally in $\S 1.2$, and to prove these results. The First Reduction Theorem is obtained in $\$ 2.2$, and the Second Reduction Theorem is proved in $\S 2.3$. The proofs of both results are fairly involved, and a number of preliminary lemmas are required. These are obtained in $\S 2.1$. The definition of elementary transformation is also given in $\$ 2.1$.

2.1 Preliminaries. All the results in this subsection refer to an $H N N$ group $G$ with base $H$, stable letter $t$, and associated subgroups $K_{-1}$ and $K_{1}$.

Lemma 4. Let $X$ and $Y$ be t-reduced words. Any one of the following statements implies one of the other two.

(i) $X$ is cyclically t-reduced.

(ii) $X Y$ is t-reduced.

(iii) $X^{-1} Y$ is t-reduced.

Proof. It can be assumed that $|X|>0$ and $|Y|>0$, for otherwise the result is obvious. Suppose $X$ has initial segment $x t^{\epsilon}$ and terminal segment $t^{\epsilon^{\prime}} x^{\prime}$, and $Y$ has initial segment $y t^{\delta} \quad(\delta= \pm 1)$. Here $x, x^{\prime}, y$ are $t$-free. Then:

$X$ is cyclically $t$-reduced unless $\epsilon=-\epsilon^{\prime}$ and $x^{\prime} x \in K_{-\epsilon}$;

$X Y$ is $t$-reduced unless $\epsilon^{\prime}=-\delta$ and $x^{\prime} y \in K_{-\delta}$;

$X^{-1} Y$ is $t$-reduced unless $\epsilon=\delta$ and $x^{-1} y \in K_{-\epsilon}$.

It is now easy to see that if any two of (i), (ii), (iii) fail then the third one does also.

The next few results concern the cyclically $t$-reduced form of a $t$-reduced word. It should be noticed that if $W$ is $t$-reduced then the cyclically $t$-reduced 
form of $W$ is the $t$-reduced form of $T^{-1} W T$, where $T$ is an initial segment of $W$ and $|T| \leqslant|W| / 2$. Moreover, $T$ can be determined algorithmically if $t$-reducing is algorithmic.

LemMA 5. Let $W$ be a t-reduced word with cyclically t-reduced form $V$, and let $T$ be the initial segment of $W$ such that $V$ is the t-reduced form of $T^{-1} W T$. If $T_{1}$ is a terminal segment of $T$ then $T_{1} V$ and $V T_{1}^{-1}$ are $t$-reduced.

Proof. Let $X$ be the $t$-reduced form of $W T$. Then the $t$-reduced form of $T^{-1} X$ is $V$, and all the $t$-symbols from $T^{-1}$ are removed in $t$-reducing $T^{-1} X$. Thus $T V$ is $t$-reduced by Lemma 2 , and consequently $T_{1} V$ is $t$-reduced.

In a similar way, if $Y$ is the $t$-reduced form of $T^{-1} W$ then the $t$-reduced form of $Y T$ is $V$ and all the $t$-symbols from $T$ are removed in $t$-reducing $Y T$. Hence $V T^{-1}$, and therefore $V T_{1}^{-1}$, are $t$-reduced. This completes the proof of the lemma.

Another point to observe is that if the cyclically $t$-reduced form of a $t$-reduced word $W$ has $t$-length greater than zero then the $t$-reduced form of $W^{n}$ $(n \neq 0)$ has an initial segment of $t$-length $[|W| / 2]+1$ identical with an initial segment of $W^{\mathrm{sg}(n)}$ and a terminal segment of $t$-length $[|W| / 2]+1$ identical with a terminal segment of $W^{\mathrm{sg}(n)}$. This is easily proved by induction.

Lemma 6. Let $X$ be a cyclically t-reduced word and $T$ a t-reduced word. Then there is a cyclically t-reduced word $X_{1}$ with $\left|X_{1}\right|=|X|$, and a terminal segment $T_{2}$ of $T$, which is either empty or has t-length greater than zero, such that the t-reduced form of $T^{-1} X T$ is $T_{2}^{-1} X_{1} T_{2}$. Moreover, if $t$-reducing is algorithmic then $X_{1}$ and $T_{2}$ are effectively calculable.

Proof. By Lemma 4 one of $T^{-1} X, X T$ is $t$-reduced. Assume for definiteness that $X T$ is $t$-reduced. Suppose that in $t$-reducing $T^{-1}(X T)$ exactly $\lambda$ $t$-symbols from $T^{-1}$ are removed. Let $T_{1}$ be the initial segment of $T$ up to but not including the $(\lambda+1)$ th $t$-symbol (if $\lambda=|T|$ take $T_{1}$ to be all of $T$ ) and let $T_{2}$ be the rest of $T$. Let $X_{1}$ be the $t$-reduced form of $T_{1}^{-1} X T_{1}$. Then

$$
\left|X_{1}\right|=\left|X T_{1}\right|-\left|T_{1}\right|=|X|+\left|T_{1}\right|-\left|T_{1}\right|=|X| .
$$

Also, by the definition of $T_{1}, T_{2}^{-1} X_{1} T_{2}$ is $t$-reduced. Clearly $X_{1}$ is cyclically $t$-reduced if $\left|X_{1}\right|=0$ or $\left|T_{1}\right|=0$. Suppose $\left|X_{1}\right|>0$ and $\left|T_{1}\right|>0$. Then $X_{1} T_{1}^{-1}$ is not $t$-reduced. However, $T_{1} X_{1}$ is $t$-reduced by Lemma 2 , and so $X_{1}$ is cyclically $t$-reduced by Lemma 4 .

The second part of the lemma is obvious.

The following transformations of pairs $\left(W_{1}, W_{2}\right)$ of $t$-reduced words will be called elementary transformations: 
(a) interchanging $W_{1}$ and $W_{2}$;

(b) replacing $W_{i}$ by $W_{i}^{\epsilon_{i}}$ for $i=1,2$;

(c) replacing $W_{i}$ by the $t$-reduced form of one of $W_{j} W_{i}^{\epsilon}, W_{i} W_{j}^{\epsilon}$ for $i \neq j$, and leaving $W_{j}$ fixed;

(d) replacing $W_{1}$ and $W_{2}$ by the $t$-reduced forms of $T^{-1} W_{1} T, T^{-1} W_{2} T$ respectively, where $T$ is a $t$-reduced word.

It is easy to see that if $\left(W_{1}^{\prime}, W_{2}^{\prime}\right)$ is obtained from $\left(W_{1}, W_{2}\right)$ by a finite sequence of elementary transformations then $\operatorname{sgp}\left\{W_{1}^{\prime}, W_{2}^{\prime}\right\}$ is conjugate to $\operatorname{sgp}\left\{W_{1}, W_{2}\right\}$. In particular, $\left(W_{1}, W_{2}\right)$ generates $G$ if and only if $\left(W_{1}^{\prime}, W_{2}^{\prime}\right)$ does.

Lemma 7. Let $W_{1}, W_{2}$ be t-reduced words which generate $G$, and suppose $\left(W_{1}^{\prime}, W_{2}^{\prime}\right)$ is obtained from $\left(W_{1}, W_{2}\right)$ by a finite sequence of elementary transformations. Then $\left(W_{1}, W_{2}\right)$ and $\left(W_{1}^{\prime}, W_{2}^{\prime}\right)$ are Nielsen equivalent.

This is easily proved by induction on the number of elementary transformations used to transform $\left(W_{1}, W_{2}\right)$ to $\left(W_{1}^{\prime}, W_{2}^{\prime}\right)$.

2.2 The first reduction theorem. In this subsection the following result will be proved.

Theorem 5. Let $G$ be an HNN group with base $H(H \neq 1)$, stable letter $t$ and associated subgroups $K_{-1}$ and $K_{1}$, and let $(X, Y)$ be a pair of t-reduced words in the generator of $G$. Then $(X, Y)$ can be transformed by a finite sequence of elementary transformations to a pair $(\widetilde{X}, \widetilde{Y})$, where $(\tilde{X}, \tilde{Y})$ generates $G$ only if $\tilde{Y} \mid=0$.

If $t$-reducing is algorithmic then there is an algorithm for obtaining for any pair $(X, Y)$ the pair $(\widetilde{X}, \widetilde{Y})$.

It is worth remarking, though the fact is not needed here, that if $|\widetilde{Y}| \neq 0$ then $\operatorname{sgp}\{\tilde{X}, \widetilde{Y}\}$ is free of rank 2 and has trivial intersection with every conjugate of the base. It should also be pointed out, though again the fact is not needed, that Theorem 5 remains true if $H=1$. In this case $G$ is the infinite cyclic group generated by $t$ and any pair of $t$-reduced words can be transformed by a finite sequence of elementary transformations to a pair of the form $\left(t^{\lambda}, 1\right)$.

Only the first part of Theorem 5 will be proved explicitly; the proof will be seen to provide the algorithm needed for the second part. The reader will notice that the proof has some similarity with the proof of Nielsen's Reduction Theorem [12, Theorem 3.1]. I have been able to extend Nielsen's theorem to $H N N$ groups, but am far from convinced that the reduction procedure can be carried out in a finite number of steps if the base group is infinite.

The idea of the proof of Theorem 5 is as follows. Since the pair $(X, Y)$ is arbitrary there is little one can say about the $t$-reduced forms of the words $X Y$, 
$X^{-1} Y, X Y^{-1}, X^{-1} Y^{-1}$. It is therefore difficult to keep a check on the $t$-reduction of a word in $X$ and $Y$. However by transforming $(X, Y)$ one can obtain a new pair $(\tilde{X}, \tilde{Y})$, with $|\widetilde{X}| \geqslant|\widetilde{Y}|$, such that the extent of $t$-reduction in the words $\widetilde{X} \widetilde{Y}, \widetilde{X}^{-1} \widetilde{Y}, \widetilde{X} \widetilde{Y}^{-1}, \widetilde{X}^{-1} \widetilde{Y}^{-1}$ is very restricted. Specifically:

(i) The words $\widetilde{X}^{-1} \widetilde{Y}, \widetilde{X} \widetilde{Y}^{-1}, \widetilde{X}^{-1} \widetilde{Y}^{-1}$ are t-reduced, and if $|\widetilde{Y}|>0$ then neither all the $t$-symbols from $\widetilde{X}$ nor all the $t$-symbols from $\widetilde{Y}$ are removed in t-reducing $\widetilde{X} \widetilde{Y}$.

Furthermore:

(ii) If $|\widetilde{Y}|>0$ then the cyclically t-reduced forms of $\tilde{X}$ and $\tilde{Y}$ have t-length greater than zero.

It follows from (i) and (ii) that if $|\widetilde{Y}|>0$ then $\operatorname{sgp}\{\tilde{X}, \tilde{Y}\} \neq G$. To prove this, let $l$ be a positive integer and let $m_{i}, n_{i}(i=1,2, \ldots, l)$ be integers, nonzero except possibly for $m_{1}$ and $n_{l}$. Assume that if $l=1$ then either $m_{1} \neq 0$ or $n_{l} \neq 0$, and let $W$ be the word $\widetilde{X}^{m}{ }^{m} \tilde{Y}^{n_{1}} \ldots \tilde{X}^{m} \tilde{Y}^{n_{l}} l$. It will be shown that if $|\widetilde{Y}|>0$ then the $t$-reduced form of $W$ has $t$-length greater than zero. Consequently, by Britton's Lemma, $W$ is not equal in $G$ to an element of $H$. Since $W$ is an arbitrary nonempty freely reduced word in $\widetilde{X}$ and $\widetilde{Y}$ it thus follows that $\operatorname{sgp}\{\tilde{X}, \tilde{Y}\} \cap H=1$, and so $\operatorname{sgp}\{\tilde{X}, \widetilde{Y}\} \neq G$.

There are two cases to consider.

Case (a). $\tilde{X} \tilde{Y}$ is t-reduced.

In order to $t$-reduce $W$ it is only necessary to $t$-reduce the subwords $\widetilde{X}^{m_{i}}$, $\widetilde{Y}^{n_{i}}$. For if $m_{i} \neq 0$ then it follows from (ii) that the $t$-reduced form of $\widetilde{X}^{m} i$ has an initial segment of $t$-length greater than zero identical with an initial segment of $\widetilde{X}^{\mathrm{sg}\left(m_{i}\right)}$, and a terminal segment of $t$-length greater than zero identical with a terminal segment of $\widetilde{X}^{\mathrm{sg}\left(m_{i}\right)}$. A similar comment applies to the $t$-reduced form of $\widetilde{Y}^{n_{i}}$ if $n_{i} \neq 0$. It is now easily seen (using (i)) that the $t$-reduced form of $W$ has $t$-length greater than zero.

Case (b). $\tilde{X} \tilde{Y}$ is not t-reduced.

Since $\widetilde{X}^{-1} \widetilde{Y}$ is $t$-reduced it follows from Lemma 4 that $\widetilde{X}$ is cyclically $t$ reduced. Similarly, since $\widetilde{Y} \widetilde{X}^{-1}$ is $t$-reduced (but $\widetilde{Y}^{-1} \widetilde{X}^{-1}$ is not), $\widetilde{Y}$ is cyclically $t$-reduced. It now follows from (i) that in order to $t$-reduce $W$ it is only necessary to choose those $i$ for which $\operatorname{sg}\left(m_{i}\right)=\operatorname{sg}\left(n_{i}\right)=1$ and replace the subword $\widetilde{X}^{\mathrm{sg}\left(m_{i}\right)} \widetilde{Y}^{\mathrm{sg}\left(n_{i}\right)}$ by the $t$-reduced form of $\widetilde{X} \widetilde{Y}$, and choose those $i$ for which

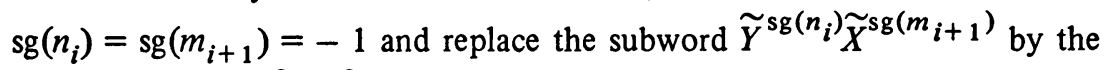
$t$-reduced form of $\tilde{Y}^{-1} \tilde{X}^{-1}$. It can now be seen that the $t$-length of the $t$-reduced form of $W$ is greater than zero.

The transformation of $(X, Y)$ to $(\tilde{X}, \tilde{Y})$ is done in three main stages. In the first stage $(X, Y)$ is transformed to a pair $\left(U_{1}, V_{1}\right)$ where $U_{1}^{-1} V_{1}$ and 
$U_{1} V_{1}^{-1}$ are $t$-reduced. In the second stage $\left(U_{1}, V_{1}\right)$ is transformed to a pair $\left(U_{2}, V_{2}\right)$ where $U_{2}^{-1} V_{2}, U_{2} V_{2}^{-1}, U_{2}^{-1} V_{2}^{-1}$ are $t$-reduced. In the third stage $\left(U_{2}\right.$, $\left.V_{2}\right)$ is transformed to a pair $\left(U_{3}, V_{3}\right)$ such that $U_{3}^{-1} V_{3}, U_{3} V_{3}^{-1}, U_{3}^{-1} V_{3}^{-1}$ are $t$-reduced, and if $\left|U_{3}\right|>0,\left|V_{3}\right|>0$ then neither all the $t$-symbols from $U_{3}$ nor all the $t$-symbols from $V_{3}$ are removed in $t$-reducing $U_{3} V_{3}$. The pair $(\tilde{X}, \tilde{Y})$ is then obtained from $\left(U_{3}, V_{3}\right)$ as follows. In the case when the cyclically $t$-reduced forms of $U_{3}$ and $V_{3}$ have $t$-length greater than zero take $(\tilde{X}, \tilde{Y})$ to be $\left(U_{3}, V_{3}\right)$ or $\left(V_{3}^{-1}\right.$, $\left.U_{3}^{-1}\right)$ according as $\left|U_{3}\right| \geqslant\left|V_{3}\right|$ or $\left|U_{3}\right|<\left|V_{3}\right|$. Otherwise, by interchanging $U_{3}$ and $V_{3}$ if necessary, it can be assumed that $V_{3}$ has an initial segment $T$ such that the $t$-reduced form of $T^{-1} V_{3} T$ has $t$-length zero. Then take $\widetilde{X}$ to be the $t$-reduced form of $T^{-1} U_{3} T$ and $\widetilde{Y}$ to be the $t$-reduced form of $T^{-1} V_{3} T$.

It will now be shown how to transform $(X, Y)$ to $\left(U_{3}, V_{3}\right)$.

Stage 1. Transformation of $(X, Y)$ to $\left(U_{1}, V_{1}\right)$.

Let $Q$ be the initial segment of $X$ such that the $t$-reduced form $X_{1}$ of $Q^{-1} X Q$ is the cyclically $t$-reduced form of $X$. Let $Y_{1}$ be the $t$-reduced form of $Q^{-1} Y Q$. Let $T$ be the initial segment of $Y_{1}$ such that the $t$-reduced form $Y_{2}$ of $T^{-1} Y_{1} T$ is the cyclically $t$-reduced form of $Y_{1}$. By Lemma 6 there is a cyclically $t$-reduced word $X_{2}$ and a terminal segment $T_{2}$ of $T$ such that the $t$-reduced form of $T^{-1} X_{1} T$ is $T_{2}^{-1} X_{2} T_{2}$. If all four words $\left(T_{2}^{-1} X_{2} T_{2}\right)^{ \pm 1} Y_{2},\left(T_{2}^{-1} X_{2} T_{2}\right)^{ \pm 1} Y_{2}^{-1}$, are $t$-reduced then take $\left(U_{1}, V_{1}\right)$ to be the pair $\left(T_{2}^{-1} X_{2} T_{2}, Y_{2}\right)$. Suppose that $\left(T_{2}^{-1} X_{2} T_{2}\right)^{\epsilon} Y_{2}^{\epsilon^{\prime}}$ is not $t$-reduced. It follows from Lemma 5 that $\left|T_{2}\right|=0$ so that both $T_{2}^{-1} X_{2} T_{2}$ and $Y_{2}$ are cyclically $t$-reduced. Consequently $\left(T_{2}^{-1} X_{2} T_{2}\right)^{-\epsilon} Y_{2}^{\epsilon^{\prime}}$ and $\left(T_{2}^{-1} X_{2} T_{2}\right)^{\epsilon} Y_{2}^{-\epsilon^{\prime}}$ are $t$-reduced, by Lemma 4. Take $\left(U_{1}, V_{1}\right)$ to be the pair $\left(\left(T_{2}^{-1} X_{2} T_{2}\right)^{\epsilon}, Y_{2}^{\epsilon^{\prime}}\right)$.

For Stages 2 and 3 the following lemma is needed. A pair $(R, S)$ of $t$-reduced words is said to be of Type 1 if $R^{-1} S$ and $R S^{-1}$ are $t$-reduced, and is said to be of Type 2 if $R^{-1} S, R S^{-1}$ and $R^{-1} S^{-1}$ are $t$-reduced. The pair is said to be of Type iA $(i=1,2)$ if it is of Type $i$ and $|R|=0$; or $|S|=0$; or $|R|>$ $0,|S|>0$ and neither all the $t$-symbols from $R$ nor all the $t$-symbols from $S$ are removed in $t$-reducing $R S$.

\section{LemMA 8. A pair of Type $i(i=1,2)$ can be transformed to a pair of} Type $i A$ by a finite sequence of elementary transformations.

Proof. Suppose $(R, S)$ is of Type $i$ but not of Type $i A$. If all the $t$-symbols from $R$ are removed in $t$-reducing $R S$ then transform $(R, S)$ to $(R, T)$, where $T$ is the $t$-reduced form of $R S$. Then clearly $R T^{-1}$ is $t$-reduced, and $R^{-1} T$ is $t$-reduced by Lemma 2. Moreover, if $i=2$ then $R^{-1} T^{-1}$ is $t$-reduced. Thus $(R, T)$ is of Type $i$. Note that $|(R, T)|<|(R, S)|$. If all the $t$-symbols from $S$, but not all the $t$-symbols from $R$, are removed in $t$-reducing $R S$ then transform 
$(R, S)$ to $(T, S)$. Then $|(T, S)|<|(R, S)|, T^{-1} S, T S^{-1}$ are $t$-reduced and $T^{-1} S^{-1}$ is $t$-reduced if $i=2$. Thus in either case $(R, S)$ is transformed to a pair $\left(R^{\prime}, S^{\prime}\right)$ of Type $i$ with $\left|\left(R^{\prime}, S^{\prime}\right)\right|<|(R, S)|$. If $\left(R^{\prime}, S^{\prime}\right)$ is not of Type $i A$ then repeat the above procedure with $\left(R^{\prime}, S^{\prime}\right)$ in place of $(R, S)$. Continuing in this way, after a finite number of steps a pair of Type $i A$ will be obtained. This completes the proof.

Stage 2. Transformation of $\left(U_{1}, V_{1}\right)$ to $\left(U_{2}, V_{2}\right)$.

By Lemma $8,\left(U_{1}, V_{1}\right)$ can be transformed to a pair $(U, V)$ of Type $1 \mathrm{~A}$. If $U V$ is $t$-reduced then take $\left(U_{2}, V_{2}\right)$ to be the pair $\left(U^{-1}, V^{-1}\right)$.

Suppose $U V$ is not $t$-reduced. Then obviously $|U|>0,|V|>0$. Also, since $U^{-1} V$ and $U V^{-1}$ are $t$-reduced it follows from Lemma 4 that $U$ and $V$ are cyclically $t$-reduced. Now suppose that in $t$-reducing $U V$ exactly $\lambda t$-symbols are removed from $U$ (and hence from $V$ ). Let $\bar{U}$ be the initial segment of $U$ up to and including the $(|U|-\lambda)$ th $t$-symbol and let $P$ be the rest of $U$. Let $Q$ be the initial segment of $V$ up to but not including the $(\lambda+1)$ th $t$-symbol and let $\bar{V}$ be the rest of $V$. Then $\bar{U}$ ends with $t^{\epsilon}$, say, and $P$ has initial segment $u t^{\delta}$ where $u$ is $t$-free and $\delta= \pm 1$; and $Q$ has terminal segment $t^{-\delta} v$ with $v t$-free, and $\bar{V}$ starts with $t^{\gamma}$ say $(\gamma= \pm 1)$. The $t$-reduced form of $P Q$ is $u k v$ where $k$ is $t$-free and belongs to $K_{-\delta}$, and $\bar{U} u k v \bar{V}$ is $t$-reduced (so that $\bar{U} u k v \bar{V}$ is the $t$-reduced form of $U V)$. Let $Z$ be the $t$-reduced form of $\bar{V} \bar{U}$. Three cases arise:

Case (i). Neither all the $t$-symbols from $\bar{V}$ nor all the $t$-symbols from $\bar{U}$ are removed in t-reducing $\bar{V} \bar{U}$ to $Z$.

Using an elementary transformation of type (c) map $(U, V)$ to $(\bar{U} P, \bar{U} u k v \bar{V})$, and then map this pair to $(P \bar{U}, u k v Z)$ by an elementary transformation of type (d) ("conjugate by $\left.\bar{U}^{\prime \prime}\right)$. Now $(u k v Z)(P \bar{U})$ is $t$-reduced (since $\bar{U} P$ is) and $(u k v Z)^{-1}(P \bar{U})^{-1}$ is $t$-reduced (since $\bar{U} u k v \bar{V}$ is). It will be shown that $(u k v Z)^{-1}(P \bar{U})$ is $t$-reduced. Note that $u k v Z$ has initial segment $u k v t^{\gamma}$ and $P \bar{U}$ has initial segment $u t^{\delta}$. Thus $(u k v Z)^{-1}(P \bar{U})$ is $t$-reduced unless $\gamma=\delta$ and $k v \in$ $K_{-\delta}$. But if $k v \in K_{-\delta}$ then $v \in K_{-\delta}$ (since $k$ does), and so $\gamma \neq \delta$, otherwise $V$ would not be $t$-reduced. Now take $\left(U_{2}, V_{2}\right)$ to be the pair $\left(u k v Z,(P \bar{U})^{-1}\right)$.

Case (ii). All the t-symbols from $\bar{U}$ are removed in t-reducing $\bar{V} \bar{U}$ to $Z$.

Transform $(U, V)$ to $(P \bar{U}, u k v Z)$ as in Case (i). Suppose $|Z|>0$. Then $(u k v Z)^{-1}(P \bar{U})^{-1}$ is $t$-reduced (since $\bar{U} u k v \bar{V}$ is), and $(u k v Z)(P \bar{U})^{-1}$ is $t$-reduced by Lemma 2. Just as in Case (i) it can be shown that $(u k v Z)^{-1}(P \bar{U})$ is $t$-reduced. If $|Z|=0$ then it is clear that $(u k v Z)^{-1}(P \bar{U})^{-1},(u k v Z)(P \bar{U})^{-1}$ and $(u k v Z)^{-1}(P \bar{U})$ are $t$-reduced. Now take $\left(U_{2}, V_{2}\right)$ to be the pair $(u k v Z, P \bar{U})$.

Case (iii). All the $t$-symbols from $\bar{V}$, but not all the $t$-symbols from $\bar{U}$, are removed in t-reducing $\bar{V} \bar{U}$ to $Z$.

This case reduces to Case (ii) by replacing $(U, V)$ by $\left(V^{-1}, U^{-1}\right)$. 
Stage 3. Transformation of $\left(U_{2}, V_{2}\right)$ to $\left(U_{3}, V_{3}\right)$.

Use the procedure of the proof of Lemma 8.

2.3 The second reduction theorem. The aim of this section is to give an improved version of Theorem 5 for $H N N$ groups in which the associated subgroups are malnormal in the base. It will be convenient to define a subgroup $A$ of a group $B$ to be proper if $A \neq 1$ and $A \neq B$.

THEOREM 6. Let $G$ be a two-generator HNN group with base $H$, stable letter $t$, and associated subgroups $K_{-1}$ and $K_{1}$, and suppose that $K_{-1}$ and $K_{1}$ are proper malnormal subgroups of $H$. Let $(X, Y)$ be a pair of $t$-reduced words in the generators of $G$. Then $(X, Y)$ can be transformed by a finite sequence of elementary transformations to a pair $(\hat{X}, \hat{Y})$, where $(\hat{X}, \hat{Y})$ generates $G$ only if $\hat{X}$ has the form tx where $x$ is $t$-free, and $\hat{Y}$ is $t$-free and defines an element of $K_{-1}$.

If t-reducing is algorithmic then there is an algorithm for obtaining for any pair $(X, Y)$ the pair $(\hat{X}, \hat{Y})$.

The following consequence of the malnormality of $K_{-1}$ and $K_{1}$ in $H$ will be used frequently throughout the proof of Theorem 6.

LeMmA 9. Let $Z$ be a t-reduced word with $|Z|>0$ and let $h$ be a $t$-free word. Suppose there is an integer $n_{0}$ such that $h^{n_{0}} \neq 1$ in $G$ and $Z^{-1} h^{n} 0 Z$ is not $t$-reduced. Then $Z^{-1} h^{n} Z$ is not t-reduced for all integers $n$.

Proof. Suppose $Z$ has initial segment $z t^{\epsilon}$ where $z$ is $t$-free. Then $z^{-1} h^{n} 0_{z}$ $\in K_{-\epsilon}$ and $z^{-1} h^{n} 0_{z} \neq 1$ in $G$. Now for any integer $n, z^{-1} h^{n} z$ commutes with $z^{-1} h^{n} 0_{z}$, and therefore lies in $K_{-\epsilon}$ by malnormality. Thus $Z^{-1} h^{n} Z$ is not $t$-reduced, and the proof is complete.

Just as for Theorem 5 only the first part of Theorem 6 will be proved explicitly; the reader will be able to obtain from this the algorithm needed for the second part.

Let $(\widetilde{X}, \widetilde{Y})$ be the pair obtained from $(X, Y)$ as in Theorem 5. It follows from Theorem 5 that if $|\widetilde{Y}|>0$ then $\operatorname{sgp}\{X, Y\} \neq G$, so in this case it suffices to take $(\hat{X}, \hat{Y})$ to be the pair $(\tilde{X}, \widetilde{Y})$.

Suppose that $|\widetilde{Y}|=0$. By considering the factor group of $G$ by the normal subgroup generated by $H$ it is not difficult to see that if the exponent sum $\tau$ of $\widetilde{X}$ on $t$ is different from 1 or -1 then $(\widetilde{X}, \widetilde{Y})$ (and therefore $(X, Y)$ ) does not generate $G$. Thus in the case when $|\widetilde{Y}|=0$ and $\tau \neq \pm 1$ it again suffices to take for $(\hat{X}, \hat{Y})$ the pair $(\tilde{X}, \widetilde{Y})$. (Note that, since $\tau \neq \pm 1,|\widetilde{X}| \neq 1$.)

Now suppose that $|\widetilde{Y}|=0$ and that $\tau= \pm 1$. Let $T$ be the initial segment of $\widetilde{X}$ such that the $t$-reduced form of $T^{-1} \tilde{X} T$ is the cyclically $t$-reduced form $U$ of $\tilde{X}$. By Lemma 6 there is a $t$-free word $h$ and a terminal segment $T_{2}$ of $T$, which is either empty or has $t$-length greater than zero, such that the $t$-reduced 
form of $T^{-1} \tilde{Y} T$ is $T_{2}^{-1} h T_{2}$. It will be shown that if $|U| \neq 1$, or $\left|T_{2}\right| \neq 0$, or both $U^{-1} T_{2}^{-1} h T_{2} U$ and $U T_{2}^{-1} h T_{2} U^{-1}$ are $t$-reduced then $\operatorname{sgp}\left\{U, T_{2}^{-1} h T_{2}\right\} \neq$ $G$, and so in any of these situations $(\hat{X}, \hat{Y})$ can be taken to be the pair $\left(U, T_{2}^{-1} h T_{2}\right)$.

Before proceeding with the proof it should be noticed that, since the exponent of $U$ on $t$ is \pm 1 , the $t$-length of $U$ is odd.

Now a word in $U$ and $T_{2}^{-1} h T_{2}$ is equal in $G$ to a word of the form

$$
U^{m_{1}} T_{2}^{-1} h^{n}{ }^{1} T_{2} U^{m_{2}} T_{2}^{-1} h^{n}{ }^{n} T_{2} \cdots U^{m}{ }^{m} T_{2}^{-1} h^{n} l T_{2},
$$

where $l>0$, the $m_{i}$ are integers, nonzero except possibly for $m_{1}$, the $n_{i}$ are integers with moduli less than the order of $h$ in $G$, and are nonzero except possibly for $n_{l}$. It will be shown that if $|U| \neq 1$, or $\left|T_{2}\right| \neq 0$, or both $U^{-1} T_{2}^{-1} h T_{2} U$ and $U T_{2}^{-1} h T_{2} U^{-1}$ are $t$-reduced, then a word $W$ as in (2.1) in which either $l>1$ or $m_{1} \neq 0$ has $t$-reduced form of $t$-length greater than zero, and therefore is not equal in $G$ to an element of $H$, by Britton's Lemma. It then follows that $\operatorname{sgp}\left\{U, T_{2}^{-1} h T_{2}\right\} \cap H \subseteq \operatorname{sgp}\left\{T_{2}^{-1} h T_{2}\right\}$, and, in particular, that $\operatorname{sgp}\left\{U, T_{2}^{-1} h T_{2}\right\}$ $\cap H$ is cyclic. Since a cyclic group has no proper malnormal subgroups $\operatorname{sgp}\left\{U, T_{2}^{-1} h T_{2}\right\} \cap H \neq H$, and so $\operatorname{sgp}\left\{U, T_{2}^{-1} h T_{2}\right\} \neq G$.

In order to show that the $t$-reduced form of $W$ has $t$-length greater than zero it is convenient to consider several cases.

Case (i). $\left|T_{2}\right|>0$.

The subwords $U^{m_{i}}$ are $t$-reduced since $U$ is cyclically $t$-reduced. It follows from Lemma 9 that the subwords $T_{2}^{-1} h^{n_{i}} T_{2}\left(n_{i} \neq 0\right)$ are $t$-reduced. Also, $T_{2} U$ and $U T_{2}^{-1}$ are $t$-reduced by Lemma 5. It now follows that in order to $t$ reduce $W$ it is only necessary to $t$-reduce the subword $T_{2}^{-1} h^{n} T_{2}$ in the case when $n_{l}=0$; and it is thus easily seen that the $t$-reduced form of $W$ has $t$-length greater than zero.

Case (ii). $\left|T_{2}\right|=0$ (that is, $T_{2}$ empty).

Subcase (ii)A. For each integer $n$ with $h^{n} \neq 1$ in $G, U h^{n} U, U^{-1} h^{n} U$, $U h^{n} U^{-1}$ are t-reduced.

Then $W$ is $t$-reduced, and $|W|>0$ since $|U|>0$.

Subcase (ii)B. For some integer $n_{0}$ with $h^{n_{0}} \neq 1$ in $G, U h^{n}{ }^{n} U$ is not t-reduced.

Since $U$ is cyclically $t$-reduced and $U\left(h^{n} 0 U\right)$ is not $t$-reduced, it follows from Lemma 4 that $U^{-1} h^{n} 0 U$ is $t$-reduced. Consequently $U^{-1} h^{n} U$ is $t$-reduced for all integers $n$ for which $h^{n} \neq 1$ in $G$, by Lemma 9. Similarly, since $U^{-1}\left(h^{-n} 0 U^{-1}\right)$ is not $t$-reduced, $U h^{n} U^{-1}$ is $t$-reduced for all $n$ for which $h^{n} \neq 1$ in $G$. Now for any integer $n$ the $t$-reduced form of $U h^{n} U$ has an initial 
segment of $t$-length at least $(|U|+1) / 2$ identical with an initial segment of $U$, and a terminal segment of $t$-length at least $(|U|+1) / 2$ identical with a terminal segment of $U$. (For the $((|U|+1) / 2)$ th $t$-symbol from the right-hand end of $U$ coincides with the $((|U|+1) / 2)$ th $t$-symbol from the left-hand end. Consequently at most $2((|U|-1) / 2) t$-symbols can be removed in $t$-reducing $U h^{n} U$.) It now follows that in order to $t$-reduce $W$ it suffices to partition the sequence $m_{1}, m_{2}, \ldots$, $m_{l}$ into "blocks"

$$
\begin{aligned}
& m_{1}, \quad m_{2}, \quad \ldots, m_{j(1)} \text {, } \\
& m_{j(1)+1}, m_{j(1)+2}, \ldots, m_{j(2)} \text {, } \\
& m_{j(s)+1}, \quad m_{j(s)+2}, \ldots, m_{l},
\end{aligned}
$$

where the $m$ 's in the same block have the same sign and $m$ 's in consecutive blocks have different signs, and then $t$-reduce the subwords

$$
\begin{array}{ll}
U^{m_{1}} h^{n_{1}} & \cdots U^{m_{j(1)} h^{n_{j(1)}},} \\
U^{m_{j(1)+1} h^{n_{j(1)+1}}} & \cdots U^{m_{j(2)} h^{n_{j(2)}},} \\
& \vdots \\
U^{m_{j(s)+1} h^{n_{j(s)+1}}} & \cdots U^{m_{l} h^{n_{l}} .}
\end{array}
$$

It is thus not difficult to see that the $t$-reduced form of $W$ has $t$-length greater than zero. t-reduced.

Subcase (ii)C. For some integer $n_{0}$ with $h^{n_{0}} \neq 1$ in $G, U^{-1} h^{n} 0 U$ is not

By Lemma $9, U^{-1} h^{n} U$ is not $t$-reduced for all integers $n$; in particular, $U^{-1} h U$ is not $t$-reduced and so $|U|>1$ (remember it is being assumed that either $|U| \neq 1$, or $\left|T_{2}\right| \neq 0$, or both $U^{-1} T_{2}^{-1} h T_{2} U, U T_{2}^{-1} h T_{2} U^{-1}$ are $t$-reduced). Since $U$ is cyclically $t$-reduced and $U^{-1}\left(h^{n} U\right)$ is not $t$-reduced it follows from Lemma 4 that $U h^{n} U$ is $t$-reduced for all integers $n$.

Now since the exponent sum of $U$ on $t$ is \pm 1 , and since $|U|>1$, it follows that the exponents on $t$ in $U$ do not all have the same sign. Thus $U$ can be partitioned into three subwords, say $U \equiv P \bar{U} Q$, where

$$
P \equiv u_{0} t^{\epsilon} u_{1} t^{\epsilon} \cdots t^{\epsilon} u_{p}, \quad Q \equiv u_{q} t^{\epsilon} u_{q+1} t^{\epsilon} \cdots t^{\epsilon} u_{s},
$$


with $u_{0}, u_{1}, \ldots, u_{p} ; u_{q}, u_{q+1}, \ldots, u_{s} t$-free, and where $\bar{U}$ has $t^{-\epsilon}$ as first and last symbol.

Now in order to $t$-reduce $U^{-1} h^{n} U\left(h^{n} \neq 1\right.$ in $\left.G\right)$ it is only necessary to $t$-reduce $P^{-1} h^{n} P$. To prove this it suffices to show that if the $t$-reduced form $V$ of $P^{-1} h^{n} P$ has $t$-length zero then $\bar{U}^{-1} V \bar{U}$ is $t$-reduced. Now if $|V|=0$ then $V \equiv$ $u_{p}^{-1} k u_{p}$, where $k$ is $t$-free and belongs to $K_{\epsilon}$, and $k \neq 1$ in $G$. Thus $\bar{U}^{-1} V \bar{U}$ is $t$-reduced unless $u_{p}^{-1} k u_{p} \in K_{\epsilon}$. But if $u_{p}^{-1} k u_{p} \in K_{\epsilon}$ then $u_{p} \in K_{\epsilon}$ (since $K_{\epsilon}$ is malnormal in $H$ and $k \in K_{\epsilon}$ ), which contradicts the fact that $U$ is $t$-reduced. In a similar way it can be shown that if $q<s$ then in order to $t$-reduce $U h^{n} U^{-1}$ $\left(h^{n} \neq 1\right.$ in $\left.G\right)$ it is only necessary to $t$-reduce $Q h^{n} Q^{-1}$. This is also true if $q=s$. To prove this it must be established that $u_{s} h^{n} u_{s}^{-1} \notin K_{-\epsilon}$. Now $u_{0}^{-1} h^{n} u_{0} \in K_{-\epsilon}$ (since $U^{-1} h^{n} U$ is not $t$-reduced) and so if $\left(u_{s} u_{0}\right) u_{0}^{-1} h^{n} u_{0}\left(u_{s} u_{0}\right)^{-1} \in K_{-\epsilon}$ then $u_{s} u_{0} \in K_{-\epsilon}$ by malnormality. This contradicts the fact that $U$ is cyclically $t$-reduced.

It now follows that after $t$-reducing $W$ the "central pieces" $\bar{U}^{ \pm 1}$ of the subwords $U^{ \pm 1}$ remain, and so the $t$-reduced form of $W$ has $t$-length greater than zero.

Subcase (ii)D. For some integer $n_{0}$ with $h^{n} 0 \neq 1$ in $G, U h^{n}{ }^{0} U^{-1}$ is not t-reduced.

An argument similar to that used in Subcase (ii)C above (replace $U$ by $U^{-1}$ ) establishes that the $t$-reduced form of $W$ has $t$-length greater than zero.

Since the above cases cover all possibilities this establishes that if $|U| \neq 1$, or $\left|T_{2}\right| \neq 0$, or both of $U^{-1} T_{2}^{-1} h T_{2} U, U T_{2}^{-1} h T_{2} U^{-1}$ are $t$-reduced then $\operatorname{sgp}\left\{U, T_{2}^{-1} h T_{2}\right\} \neq G$.

To complete the proof of the theorem the situation where $|U|=1,\left|T_{2}\right|=$ 0 and one of $U^{-1} T_{2}^{-1} h T_{2} U, U T_{2}^{-1} h T_{2} U^{-1}$ is not $t$-reduced must be considered. By inverting $U$ if necessary it can be assumed that $t$ occurs to exponent 1 in $U$. Then $U \equiv d t g$ where $d$ and $g$ are $t$-free words. Moreover, by applying an elementary transformation of type (d) ("conjugate by $d t$ ") if necessary, it can be assumed that $U h U^{-1}$ is not $t$-reduced. Then $U h U^{-1}$ has $t$-reduced form $d k d^{-1}$, where $k$ is $t$-free and belongs to $K_{-1}$. Then take $(\hat{X}, \hat{Y})$ to be the pair $(\operatorname{tgd}, k)$.

3. Determination of FE groups. The aim of this section is to verify that various groups are FE or AFE groups. The first subsection concerns one-relator groups with torsion, the second subsection deals with small cancellation groups, and the third subsection concerns triangle groups.

3.1 One-relator groups with torsion. In this subsection the following result will be proved.

Let $H=\left\langle a, b ; P^{n}\right\rangle$ where $P$ is a cyclically reduced word involving $a$ and $b$, and $n>1$. Suppose that all the exponents to which a occurs in $P$ have the same sign, and that if $n=2$ then all the exponents to which $b$ occurs in $P$ also 
have the same sign. Then $H$ is a special $A F E$ group relative to $(a, b)$.

The proof, of course, consists of showing that Conditions 1-8 are satisfied. Conditions 1 and 5 hold by the Freiheitssatz. Conditions 3 and 8 hold by Lemma 2.1 of [21]. Condition 7 holds by Theorem 4.14 of [12] (see also Corollary 1 of [20]).

Condition 2. Let $\lambda$ be an integer. It will be shown that $\operatorname{sgp}\left\{a^{\lambda}, b^{\lambda}\right\} \cap$ $\operatorname{sgp}\{a\}=\operatorname{sgp}\left\{a^{\lambda}\right\}$. The fact that $\operatorname{sgp}\left\{a^{\lambda}, b^{\lambda}\right\} \cap \operatorname{sgp}\{b\}=\operatorname{sgp}\left\{b^{\lambda}\right\}$ is proved similarly. The result is trivial if $\lambda=0, \pm 1$, so assume $|\lambda|>1$. Let $W$ be a freely reduced word in $a^{\lambda}, b^{\lambda}$ which involves $b$ and defines an element $a^{\alpha}$ of $\operatorname{sgp}\{a\}$. Then it follows from Statement 1 on p. 1439 of [8] that when $\mathrm{Wa}^{-\alpha}$ is freely reduced it has a subword of the form $T^{n-1} T_{1}$, where $T$ is a cyclic permutation of $P$ or $P^{-1}, T \equiv T_{1} T_{2}$ and $T_{1}$ involves both $a$ and $b$, and it can easily be deduced from this that there is a cyclic permutation $S$ of $P$ with the property that all the exponents to which $a$ occurs in $S$ and all the exponents to which $b$ occurs in $S$ are divisible by $\lambda$. Thus the factor group of $H$ by the normal subgroup generated by $\left\{a^{\lambda}, b^{\lambda}\right\}$ is the free product $L=\left\langle a, b ; a^{\lambda}, b^{\lambda}\right\rangle$. Now $W=1$ in $L$, and so $a^{\alpha}=1$ in $L$. Thus $\lambda$ divides $\alpha$ as required.

Condition 4. Let $h$ be a word in $a, b$ and suppose that $a$ and $h^{-1} b h$ generate $H$. Then it is required to show that $h$ is expressible in the form $b^{\beta} a^{\alpha}$. To this end consider the set

$V=\left\{v: v\right.$ is a word in $a$ and $b$, and $b^{\kappa} v a^{\omega}$ is equal to $h$ in $H$

for suitable integers $k$ and $\omega$,

and let $w$ be an element of $V$ of minimal length. Suppose $w$ is nonempty. It will be shown that $\operatorname{sgp}\left\{a, w^{-1} b w\right\}$ is free of rank 2 . Consequently $\operatorname{sgp}\left\{a, h^{-1} b h\right\}$ is also free of rank 2 and is therefore not equal to $H$. It is important to note that the minimality of $w$ implies that the following holds.

(3.1) $w$ starts with $a$ or $a^{-1}$ and ends with $b$ or $b^{-1}, w$ does not contain more than half a cyclic permutation of $P^{n}$ or its inverse, if $n=3$ then for no integer $\gamma$ does wa ${ }^{\gamma}$ have a terminal segment identical with a cyclic permutation of $P^{ \pm(n-1)}$, if $n=2$ then $w$ is not half of any cyclic permutation of $P^{ \pm n}$.

Let $l$ be a positive integer and let $\xi_{i}, \eta_{i}(i=1,2, \ldots, l)$ be nonzero integers. Let $Q$ be the word $a^{\xi_{1}} w^{-1} b^{\eta_{1}} w \cdots a^{\xi} w^{-1} b^{\eta} l_{w}$. Observe that, as a word in $a$ and $b, Q$ is freely reduced. Consequently, by Statement 1 on p. 1439 of [8], $Q$ is not equal to 1 in $H$ provided it has no subword of the form $T^{n-1} T_{1}$, where $T$ is a cyclic permutation of $P$ or $P^{-1}, T \equiv T_{1} T_{2}$ and $T_{1}$ involves $a$ and $b$. Now no subword of $Q$ involving $a$-symbols from a subword $w$ and a subword $w^{-1}$ can be identical with a subword of a cyclic permutation of $P^{n}$ or $P^{-n}$, for all the exponents to which $a$ occurs in $P$ have the same sign. Moreover, if $n=2$ then 
neither can a subword of $Q$ involving $b$-symbols from a subword $w$ and a subword $w^{-1}$ be identical with a subword of a cyclic permutation of $P^{ \pm n}$. Taking (3.1) into account it follows that the only subwords of $Q$ involving $a$ and $b$ which could have the form $T^{n-1} T_{1}$ are of one of the following types:

$\left(v a^{p} b^{s}\right)^{\epsilon}, p \neq 0, v$ a nonempty terminal segment of $w, s=0$ if $n=2$;

$\left(b^{q} w a^{p} b^{s}\right)^{\epsilon}, p \neq 0, s=0$ if $n=2$;

$\left(b^{q} u\right)^{\epsilon}, q \neq 0, u$ a nonempty initial segment of $w$.

It therefore suffices to show that none of the above words can have the form $T^{n-1} T_{1}$. Since the inverse of a word of the form $T^{n-1} T_{1}$ is of the same form, it is enough to consider those cases when $\epsilon=1$.

Suppose $v a^{p} b^{s} \equiv T^{n-1} T_{1}$, where $T$ is a cyclic permutation of $P^{ \pm 1}, T \equiv$ $T_{1} T_{2}$ and $T_{1}$ involves $a$ and $b$. Assume first that $a^{p} b^{s}$ is a proper terminal seg. ment of $T_{1}$, so that $T_{1} \equiv T_{1}^{\prime} a^{p} b^{s}$ say, where $T_{1}^{\prime}$ is nonempty. Then $v \equiv$ $T^{n-1} T_{1}^{\prime}$. But this is impossible by (3.1). Now assume $a^{p} \equiv a^{\gamma} a^{r}$ with $r \neq 0$ and $T_{1} \equiv a^{r} b^{s}$. Then $n>2$ (since $s=0$ if $\left.n=2\right)$ and $v a^{\gamma} \equiv T^{n-1}$, and once again this is impossible by (3.1).

Next suppose $b^{q} w a^{p} b^{s} \equiv T^{n-1} T_{1}$. Then $T_{1} \equiv b^{q} T_{1}^{\prime} a^{p} b^{s}$ and so $w \equiv$ $\left(T_{1}^{\prime} a^{p} b^{s} T_{2} b^{q}\right)^{n-1} T_{1}^{\prime}$. Thus if $T_{1}^{\prime}$ is nonempty, $w$ is identical with more than half a cyclic permutation of $P^{n}$ or its inverse, and if $T_{1}^{\prime}$ is empty, $w$ has the form $S^{n-1}$ where $S$ is a cyclic permutation of $P^{ \pm 1}$. However both of these possibilities contradict (3.1).

Finally, the fact that $b^{q} u$ does not have the form $T^{n-1} T_{1}$ is verified by considerations similar to iilose used above.

Condition 6 . This follows fairly easily from the verification above that Condition 2 holds. It is not difficult to see that there is an algorithm for obtaining for any word $h$ in $a, b$ a word $b^{\kappa} w a^{\omega}$ equal to $h$ in $H$ with $w$ either empty or satisfying (3.1). Then by what has been shown previously, $h$ can be expressed in the form $b^{\beta} a^{\alpha}$ if and only if $w$ is empty.

3.2 Small cancellation groups. The aim of this subsection is to establish the following theorem.

Let $H=\left\langle a, b ; R_{1}, R_{2}, \ldots\right\rangle$ and assume that $H$ satisfies the small cancellation condition $C^{\prime}(1 / 18)$. Suppose that for each relator $R_{i}$ either all the exponents to which a occurs in $R_{i}$ have the same sign or all the exponents to which $b$ occurs in $R_{i}$ have the same sign, and that adjacent exponents in $R_{i}$ are coprime. Assume further that $a^{\kappa}$ is a relator if and only if $b^{\kappa}$ is. Then it is a special $F E$ group relative to $\left(a^{\xi}, b\right)$, where $\xi$ is coprime to the order of $a$. If the number of relators of $H$ is finite then $H$ is in fact a special AFE group relative to $\left(a^{\xi}, b\right)$.

The definition of adjacent exponents was given in $\S 1.3$.

The proof of the above theorem uses Corollary 4.1(i) of [11]. It is 
worth remarking that if one supposes that each relator is either a positive or negative word then $C^{\prime}(1 / 18)$ can be replaced by $C^{\prime}(1 / 8)$ in the above. For then $H$ satisfies the triangle condition $T_{3}$ and so Corollary 4.1(ii) of [11] is applicable. (See Theorem 2 of [22].)

Before embarking on the main part of the proof it is convenient to treat two special cases separately. Firstly, if $H=\langle a, b ; a, b\rangle$ then the result is trivial.

Secondly, suppose $H$ has a relator of length less than 18 involving $a$ and $b$. Then it is not difficult to show that then $H=\left\langle a, b ;\left(a b^{\delta}\right)^{q}\right\rangle$ where $|\delta|=1$ and $q$ is an integer with $1 \leqslant q \leqslant 8$. Then if $q=1$ the result is trivial, and if $q>1$ the result follows from the previous subsection. From now on it will be assumed that $H$ does not have a relator of length 1 and that every relator of $H$ involving $a$ and $b$ has length greater than 17 . This assumption will not be repeated.

It will now be shown that $H$ satisfies Conditions 1-4 and Condition 8 .

Condition 1. Suppose first that each relator of $H$ involves both $a$ and $b$. Then $a$ and $b$ have infinite order. For suppose that $a^{m}=1$ in $H$ where $m>0$. Then it follows from Corollary 4.1(i) of [11] that $a^{m}$ has a subword $a^{r}$ which is more than $5 / 6$ of a cyclic permutation of a relator $R_{i}$ or its inverse. Since $R_{i}$ involves $b, a^{r-1}$ is a piece and so $r-1<L\left(R_{i}\right) / 18$. However $\left.r-1\right\rangle$ $5 L\left(R_{i}\right) / 6-1$, and so $L\left(R_{i}\right)=1$ which is a contradiction. In the same way $b^{m}$ $\neq 1$ for every positive integer $m$.

Now suppose that $a^{\kappa}$ and $b^{\kappa}$ are relators, where it may be assumed that $\kappa>$ 0 . By the small cancellation condition every other relator involves $a$ and $b$. Suppose that $m$ is the order of $a$. Then $\kappa$ must be a multiple of $m$, say $\kappa=\lambda m$. Now $a^{m}$ has a subword which is more than $5 / 6$ of a cyclic permutation of a relator $R_{i}$ or its inverse, and an argument similar to that in the previous paragraph shows that $R_{i} \equiv a^{\kappa}$. Thus $\lambda=1$. In a similar way, $\kappa$ is the order of $b$ in $H$.

In the following $\gamma$ will denote the order of $a$ and $b$ in $H$. Note that, by what has been shown above,

(3.2) $\gamma$ is infinite if each relator of $H$ involves $a$ and $b ; \gamma$ is finite and equal to $|k|$ if $a^{\kappa}$ is a relator.

Condition 2. It will be shown that for every integer $\lambda, \operatorname{sgp}\left\{a^{\lambda}, b^{\lambda}\right\} \cap$ $\operatorname{sgp}\{a\}=\operatorname{sgp}\left\{a^{\lambda}\right\}$. The fact that $\operatorname{sgp}\left\{a^{\lambda}, b^{\lambda}\right\} \cap \operatorname{sgp}\{b\}=\operatorname{sgp}\left\{b^{\lambda}\right\}$ is proved similarly. The result is trivial if $\lambda=0$, or if $\lambda$ is coprime to the order of $a$, so assume $\lambda \neq 0$ and that the highest common factor $\mu$ of $\lambda$ and $\gamma$ is greater than 1 . Then it suffices to show that $\operatorname{sgp}\left\{a^{\mu}, b^{\mu}\right\} \cap \operatorname{sgp}\{a\}=\operatorname{sgp}\left\{a^{\mu}\right\}$.

Let $a^{\xi_{1}} b^{\eta_{1}} \cdots a^{\xi_{l}} b^{\eta_{l}}$ be a typical element of $\operatorname{sgp}\left\{a^{\mu}, b^{\mu}\right\}$-where $l>0$, the $\xi_{i}$ and $\eta_{i}$ are integers, nonzero except possibly for $\xi_{1}$ and $\eta_{l},\left|\xi_{i}\right|,\left|\eta_{i}\right| \leqslant$ $\gamma / 2$, the $\xi_{i}$ and $\eta_{i}$ are divisible by $\mu$-and suppose $a^{\xi_{1}} b^{\eta_{1}} \cdots a^{\xi_{l}} b^{\eta_{l}} \in \operatorname{sgp}\{a\}$. Then an equation $a^{\alpha} b^{\eta_{1}} \cdots a^{\xi_{l}} b^{\eta_{l}}=1$ takes place in $H$. Here $|\alpha| \leqslant \gamma / 2$. Let 
$Q$ denote the word on the left-hand side of this equation. Then it suffices to show that $Q$ is empty.

Suppose, by way of contradiction, that $Q$ is nonempty. Then it follows from (3.2) and Corollary 4.1(i) of [11] that $Q$ has a subword involving $a$ and $b$ which is more than $5 / 6$ of a cyclic permutation $R_{i}^{*}$ of a relator or its inverse. Since adjacent exponents in each relator are coprime the only possible subwords of $Q$ involving $a$ and $b$ which could be more than $5 / 6$ of $R_{i}^{*}$ have the form $\left(a^{p} b^{q} a^{r} b^{5}\right)^{\epsilon}$. Suppose that $\left(a^{p} b^{q} a^{r} b^{s}\right)^{\epsilon}$ is a subword of $R_{i}^{*}$ and

$$
|p|+|q|+|r|+|s|>5 L\left(R_{i}^{*}\right) / 6 .
$$

This implies that one of $|p|,|q|,|r|,|s|$ is greater than $5 L\left(R_{i}^{*}\right) / 24$. Assume for example that $|p|>5 L\left(R_{i}^{*}\right) / 24$. Since $a^{|p|-1}$ is a piece and $H$ satisfies $C^{\prime}(1 / 18)$, $|p|-1<L\left(R_{i}^{*}\right) / 18$. Consequently $L\left(R_{i}^{*}\right)<7$, which is a contradiction.

Conditions $3,4,8$. The verifications that these conditions hold can be done simultaneously. Suppose $h$ is a word in $a$ and $b$ for which one of the following equations holds in $H$.

$$
\begin{gathered}
h^{-1} a^{m} h a^{n}=1, \\
h^{-1} b^{m} h b^{n}=1, \\
a^{\xi_{1}} h^{-1} b^{\eta_{1}} h \cdots a^{\xi} h^{-1} b^{\eta_{l} h=1 .}
\end{gathered}
$$

Here $l$ is a positive integer and $m, n, \xi_{1}, \eta_{1}, \ldots, \xi_{l}, \eta_{l}$ are nonzero integers with moduli less than or equal $\gamma / 2$. It will be shown that if (3.3) holds then $h \in$ sgp $\{a\}$, if (3.4) holds then $h \in \operatorname{sgp}\{b\}$, and if (3.5) holds then $h$ is equal in $H$ to a word of the form $b^{\beta} a^{\alpha}$. The first two conclusions imply that sgp $\{a\}$ and $\operatorname{sgp}\{b\}$ are malnormal in $H$; the third conclusion implies that $\operatorname{sgp}\left\{a, h^{-1} b h\right\}$ is the free product of $\operatorname{sgp}\{a\}$ and $\operatorname{sgp}\left\{h^{-1} b h\right\}$ unless $h=b^{\beta} a^{\alpha}$ in $H$, and from this it can be deduced that Condition 4 holds. It also follows from this third conclusion that Condition 8 holds. For if $a^{m} h^{-1} b^{n} h=1$ in $H$ where $a^{m} \neq 1$ and $b^{n} \neq 1$ then $h=b^{\beta} a^{\alpha}$ in $H$, so that $a^{m}=b^{-n}$. Consequently $H$ has a centre, contradicting Theorem XI(i) of [7].

Obtain from $h$ a word equal to $h$ in $H$ which is freely reduced and does not contain more than half a cyclic permutation of a relator or its inverse. Replace $h$ in (3.3), (3.4) or (3.5) by this new word and cyclically reduce the word on the left-hand side of the equation. Then according as to whether (3.3), (3.4) or (3.5) holds one of the following is obtained.

$$
\begin{aligned}
& w_{3}^{-1} a^{m} w_{3} a^{n}=1, \\
& w_{4}^{-1} b^{m} w_{4} b^{n}=1,
\end{aligned}
$$




$$
a^{\xi} w_{5}^{-1} b^{\eta} w_{5} \cdots a^{\xi_{5}} w_{5}^{-1} b^{\eta} l_{w_{5}}=1 .
$$

Here $w_{3}$ is either empty or starts and ends with a $b$-symbol, $w_{4}$ is either empty or starts and ends with an $a$-symbol, $w_{5}$ is empty or starts with an $a$-symbol and ends with a $b$-symbol. To complete the proof it suffices to show that if $w_{i}$ ( $i=$ $3,4,5)$ is nonempty then equation (3.i') is impossible.

Suppose, by way of contradiction, that $w_{i}$ is nonempty and let $Q_{i}$ denote the word on the left-hand side of (3.i'). Since $Q_{i}$ is equal to 1 in $H$ it follows from (3.2) and Corollary 4.1(i) of [11] that $Q_{i}$ has a subword involving $a$ and $b$ which is more than $5 / 6$ of a cyclic permutation $R_{j}^{*}$ of a relator or its inverse. Since either all the exponents to which $a$ occurs in $R_{j}^{*}$ have the same sign or all the exponents to which $b$ occurs in $R_{j}^{*}$ have the same sign, the only possible subwords of $Q_{i}$ involving $a$ and $b$ which could be more than $5 / 6$ of $R_{j}^{*}$ have the form $\left(x^{p} v x^{q} y^{r} x^{s}\right)^{\epsilon}$. Here $v$ is a subword of $w_{i}$ or $w_{i}^{-1} ; x \in\{a, b\}$ and $y \in\{a, b\} \backslash\{x\}$; $p, q, r, s$ are integers at least one of which is zero.

Suppose $\left(x^{p} v x^{q} y^{r} x^{s}\right)^{\epsilon}$ is a subword of $R_{j}^{*}$ and $L\left(x^{p} v x^{q} y^{r} x^{s}\right)>5 L\left(R_{j}^{*}\right) / 6$. Since $L(v) \leqslant L\left(R_{j}^{*}\right) / 2$ it follows that $|p|+|q|+|r|+|s|>L\left(R_{j}^{*}\right) / 3$. Consequently at least one of $|p|,|q|,|r|,|s|$ is greater than $L\left(R_{j}^{*}\right) / 9$ (remembering that one of $p, q, r, s$ is zero). Suppose, for example, that $|p|>L\left(R_{j}^{*}\right) / 9$. Since $x^{|p|-1}$ is a piece and $H$ satisfies $C^{\prime}(1 / 18),|p|-1<L\left(R_{j}^{*}\right) / 18$. Hence $L\left(R_{j}^{*}\right)<$ 18 , which is a contradiction. This completes the verification that Conditions 3,4 and 8 hold.

To finish the proof of the theorem it must be shown that if $H$ is finitely presented then Conditions 5, 6, 7 hold. That Condition 5 holds follows from the verification of Condition 1. The fact that Conditions 6 and 7 hold follows from the verification of Conditions 3 and 4 . For observe that if $H$ has finitely many relators then equation $\left(3 . i^{\prime}\right)(i=3,4,5)$ is obtained algorithmically from equation (3.i). Then $h \in \operatorname{sgp}\{a\}$ if and only if $w_{3}$ is empty, $h \in \operatorname{sgp}\{b\}$ if and only if $w_{4}$ is empty, and $h$ is expressible in the form $b^{\beta} a^{\alpha}$ if and only if $w_{5}$ is empty.

3.3 Triangle groups. It will be shown in this subsection that if $H=$ $\left\langle a, b ; a^{p}, b^{p},(a b)^{q}\right\rangle$ where $2 / p+1 / q<1$ then $H$ is an $F E$ group relative to $\left(a^{\xi}, b\right)$, where $\xi$ is coprime to $p$; moreover, if $p \geqslant 4$ then $H$ is an AFE group. (4) It should be mentioned that this result follows for a large number of values of the pair $(p, q)$ from the theorem of the previous subsection.

Conditions 1, 5. Both $a$ and $b$ have order $p$ [10, Corollary 2].

Condition 2. If $\lambda$ is a nonzero integer then $\operatorname{sgp}\left\{a^{\lambda}, b^{\lambda}\right\}=H$ if $\lambda$ is coprime to $p$, and $\operatorname{sgp}\left\{a^{\lambda}, b^{\lambda}\right\}=\operatorname{sgp}\left\{a^{\lambda}\right\} * \operatorname{sgp}\left\{b^{\lambda}\right\}$ otherwise. In either case, it follows easily that $\operatorname{sgp}\left\{a^{\lambda}, b^{\lambda}\right\} \cap \operatorname{sgp}\{a\}=\operatorname{sgp}\left\{a^{\lambda}\right\}$ and $\operatorname{sgp}\left\{a^{\lambda}, b^{\lambda}\right\} \cap \operatorname{sgp}\{b\}=$ $\operatorname{sgp}\left\{b^{\lambda}\right\}$.

$\left.{ }^{4}\right)$ I thank Dr. G. Rosenberger for helping me with this subsection. 
Conditions 3, 8. See Corollary 2 of [10].

Condition 4. A proof that this holds is indicated on pp. 241-242 of [26].

Conditions 6, 7. Suppose $p \geqslant 4$. Then $H$ satisfies $C(4)$ and $T_{3}$ [11], and so the word problem for $H$ is solvable, by Theorem II of [11]. Since $a$ and $b$ have finite order it follows that Conditions 6 and 7 hold.

Added In Proof (MAY 19, 1975). With regard to the problem raised in the third from the last paragraph on p. 336, the author has recently established that if $G=\left\langle a, t ; R^{n}\right\rangle$, where $\left.n\right\rangle 1$, and where $R$ is not a power of a primitive, then $G$ has one Nielsen equivalence class.

The problem of $\mathrm{McCool}$ and Pietrowski mentioned on p. 343 has been solved affirmatively by $\mathrm{A}$. M. Brunner.

\section{REFERENCES}

1. G. Baumslag, Residually finite one-relator groups, Bull. Amer. Math. Soc. 73 (1967), 618-620. MR 35 \#2953.

2. - Some problems on one-relator groups, Proc. Second Internat. Conf. on Theory of Groups (Canberra, 1973), Lecture Notes in Mathematics, no. 372, Springer-Verlag, Berlin and New York, 1974.

3. J. L. Britton, The word problem, Ann. of Math. (2) 77 (1963), 16-32. MR 29 \#5891.

4. A. M. Brunner, Transitivity-systems of certain one-relator groups, Proc. Second Internat. Conf. on Theory of Groups (Canberra, 1973), Lecture Notes in Mathematics, no. 372, Springer-Verlag, Berlin and New York, 1974.

5. M. J. Dunwoody, On T-systems of groups, J. Austral. Math. Soc. 3 (1963), 172-179. MR 27 \#3706.

6. M. J. Dunwoody and A. Pietrowski, Presentations of the trefoil group, Canad. Math. Bull. 16 (1973), 517-520.

7. M. Greendlinger, On Dehn's algorithms for the conjugacy and word problems, with applications, Comm. Pure Appl. Math. 13 (1960), 641-677. MR 23 \#A2327.

8. G. A. Gurevič, On the conjugacy problem for groups with one defining relation, Dokl. Akad. Nauk SSSR 207 (1972), 18-20 = Soviet Math. Dokl. 13 (1972), 1436-1439.

9. G. Higman, B. H. Neumann and Hanna Neumann, Embedding theorems for groups, J. London Math. Soc. 24 (1949), 247-254. MR 11, 322.

10. A. H. M. Hoare, A. Karrass and D. Solitar, Subgroups of infinite index in Fuchsian groups, Math. Z. 125 (1972), 59-69. MR 45 \#2029.

11. R. C. Lyndon, On Dehn's algorithm, Math. Ann. 166 (1966), 208-228. MR 35 \#5499.

12. W. Magnus, A. Karrass and D. Solitar, Combinatorial group theory: Presentations of groups in terms of generators and relations, Pure and Appl. Math., vol. 13, Interscience, New York, 1966. MR 34 \#7617.

13. J. McCool and A. Pietrowski, On free products with amalgamation of two infinite cyclic groups, J. Algebra 18 (1971), 377-383. MR 43 \#6296.

14. C. F. Miller III, On Britton's Theorem A, Proc. Amer. Math. Soc. 19 (1968), 1151-1154. MR 38 \#244.

15. D. I. Moldavanskin, Certain subgroups of groups with one defining relation, Sibirsk. Mat. Ž. 8 (1967),1370-1384 = Siberian Math. J. 8 (1967), 1039-1084. MR 36 \#3862.

16. B. H. Neumann, On the number of generators of a free product, J. London Math. Soc. 18 (1943), 12-20. MR 5, 58. 
17. An essay on free products of groups with amalgamations, Philos. Trans. Roy. Soc. London Ser. A 246 (1954), 503-554. MR 16, 10.

18. - On a question of Gashütz, Arch. Math. 7 (1956), 87-90.

19. B. H. Neumann and Hanna Neumann, Zwei Klassen charakteristischer Untergruppen. und ihrer Faktorgruppen, Math. Nachr. 4 (1951), 106-125. MR 12,671.

20. B. B. Newman, Some results on one relator groups, Bull. Amer. Math. Soc. 74 (1968), 568-571. MR 36 \#5204.

21. The soluble subgroups of a one relator group with torsion, J. Austral. Math. Soc. 16 (1973), 278-285.

22. S. J. Pride, On the Nielsen equivalence of pairs of generators in certain $H N N$ groups, Proc. Second Internat. Conf. on Theory of Groups (Canberra, 1973), Lecture Notes in Mathematics, no. 372, Springer-Verlag, Berlin and New York, 1974.

23. N. Purzitsky and G. Rosenberger, Two generator Fuchsian groups of genus one, Math. Z. 128 (1972), 245-251; Correction, ibid. 132 (1973), 261-262. MR 47 \#2055; 48 \#6270.

24. E. S. Rapaport, Note on Nielsen transformations, Proc. Amer. Math. Soc. 10 (1959), 228-235. MR 21 \#3477.

25. G. Rosenberger, Automorphismen und Erzeugende für Gruppen mit einen definierenden Relation, Math. Z. 129 (1972), 259-267. MR 47 \#3552.

26. —— Eine Bemerkung zu den Triangel-Gruppen, Math. Z. 132 (1973), 239244.

27. W. W. Boone, F. B. Cannonito and R. C. Lyndon (Editors), Word problems (decision problems and the Burnside Problem in group theory), North-Holland, Amsterdam, 1973.

DEPARTMENT OF MATHEMATICS, INSTITUTE OF ADVANCED STUDIES, AUSTRALIAN NATIONAL UNIVERSITY, CANBERRA, AUSTRALIA

FACULTY OF MATHEMATICS, THE OPEN UNIVERSITY, WALTON HALL, MILTON KEYNES, BUCKINGHAMSHIRE, ENGLAND 